\title{
On Asymptotic Stability of Solitary Waves in Schrödinger Equation Coupled to Nonlinear Oscillator
}

\author{
V. S. Buslaev ${ }^{1}$ \\ Department of Mathematical and Computational Physics \\ Faculty of Physics \\ St-Petersburg University, Russia \\ e-mail: buslaev@mph.phys.spbu.ru \\ A. I. Komech ${ }^{2,3}$ \\ Fakultät für Mathematik, Universität Wien \\ Nordbergstrasse 15, Wien A-1090, Austria \\ e-mail: alexander.komech@univie.ac.at \\ E. A. Kopylova ${ }^{3}$ \\ Institute of the Information Transmission Problems $R A S$, \\ B.Karetnii per.19, Moscow 101447, Russia. \\ e-mail: ek@vpti.vladimir.ru \\ D. Stuart ${ }^{4}$ \\ Centre for Mathematical Sciences, \\ Wilberforce Road, Cambridge, CB3 OWA \\ e-mail: D.M.A.Stuart@damtp.cam.ac.uk
}

\begin{abstract}
The long-time asymptotics is analyzed for finite energy solutions of the 1D Schrödinger equation coupled to a nonlinear oscillator. The coupled system is invariant with respect to the phase rotation group $U(1)$. For initial states close to a solitary wave, the solution converges to a sum of another solitary wave and dispersive wave which is a solution to the free Schrödinger equation. The proofs use the strategy of Buslaev-Perelman $[2,3]$ : the linerization of the dynamics on the solitary manifold, the symplectic orthogonal projection, method of majorants, etc.
\end{abstract}

\footnotetext{
${ }^{1}$ Supported partly by RFBR research grants 05-0101076 and 05-01002944.

${ }^{2}$ On leave Institute of the Information Transmission Problems RAS. Supported partly by Alexander von Humboldt Research Award, RFBR grant 07-01-00018a, and Max-Planck Institute for Mathematics in the Sciences (Leipzig).

${ }^{3}$ Supported partly by FWF grant P19138-N13, DFG grant 436 RUS 113/929/0-1, and RFBR grant 06-0100096.

${ }^{4}$ Partially supported by EPSRC grant A00133/01
} 


\section{Introduction}

Our main goal is the study of the distinguished dynamical role of the "quantum stationary states" for a model $U(1)$-invariant nonlinear Schrödinger equation

$$
i \dot{\psi}(x, t)=-\psi^{\prime \prime}(x, t)-\delta(x) F(\psi(0, t)), \quad x \in \mathbb{R} .
$$

Here $\psi(x, t)$ is a continuous complex-valued wave function and $F$ is a continuous function, the dots stand for the derivatives in $t$ and the primes in $x$. All derivatives and the equation are understood in the distribution sense. Physically, equation (1.1) describes the system of the free Schrödinger equation coupled to an oscillator attached at the point $x=0: F$ is a nonlinear "oscillator force".

We assume that $F(\psi)=-\nabla U(\psi)$ where $U(\psi)=u(|\psi|)$. Then (1.1) defines a $U(1)$-invariant Hamilton system and admits finite energy solutions of type $\psi_{\omega}(x) e^{i \omega t}$ called solitary waves or nonlinear eigenfunctions. The solitary waves constitute a two-dimensional solitary manifold in the Hilbert phase space of finite energy states of the system. We prove the asymptotics of type

$$
\psi(\cdot, t) \sim \psi_{\omega_{ \pm}} e^{i \omega_{ \pm} t}+W(t) \Phi_{ \pm}, \quad t \rightarrow \pm \infty
$$

where $W(t)$ is the dynamical group of the free Schrödinger equation, $\Phi_{ \pm} \in C_{b}(\mathbb{R}) \cap L^{2}(\mathbb{R})$ are the corresponding asymptotic scattering states, and the remainder converges to zero as $\mathcal{O}\left(|t|^{-1 / 2}\right)$ in global norm of $C_{b}(\mathbb{R}) \cap L^{2}(\mathbb{R})$. Here $C_{b}(\mathbb{R})$ is the space of bounded continuous functions $\mathbb{R} \rightarrow \mathbb{C}$. The asymptotics hold for the solutions with initial states close to the stable part of the solitary manifold, extending the results of $[2,3,16,18,19]$ for different one-dimensional equations to equation (1.1).

Let us note that we impose conditions which are more general than the standard ones in the following respects:

i) We do not hypothesize any spectral properties of the linearized equation, and do not require any smallness condition on the initial state (only closeness to the solitary manifold).

ii) The stable part of the solitary manifold is characterized by a condition on the nonlinearity (3.2). The relation of this to the standard criterion for orbital stability $\partial_{\omega} \int\left|\psi_{\omega}(x)\right|^{2} d x>0$ (see [9] and references therein) will be discussed below.

This progress is possible owing to the simplicity of our model which allows us to obtain explicit formula for the resolvent and derive all spectral properties of the linearization using this formula. In higher dimensions the explicit formula for the resolvent is missing hence similar exact analysis of spectral properties is impossible.

Our investigation is inspired by a fundamental problem of quantum mechanics. The solitary waves were introduced by Schrödinger for the quantum electron coupled to the Maxwell field, [23]. He identified the solitary waves with the quantum stationary states. The asymptotics of type (1.2) for the coupled U(1)-invariant Maxwell-Schrödinger equations would describe the transitions between the quantum stationary states, while the dispersive wave $W(t) \Phi_{ \pm}$would correspond to the electromagnetic radiation. Let us note however, that the asymptotics of type (1.2) are not proved yet for the coupled equations.

For the first time, the asymptotics of type (1.2) were established by Soffer and Weinstein $[24,25]$ (see also [20]) for nonlinear $U(1)$-invariant Schrödinger equation with small initial states if the nonlinear coupling constant is sufficiently small. The next result was obtained by Buslaev and Perelman $[2,3]$ who proved that the solitary manifold attracts finite energy solutions of 
a 1D nonlinear $U(1)$-invariant translation invariant Schrödinger equation with initial states sufficiently close to the stable part of the solitary manifold.

The novel techniques of Buslaev and Perelman are based on the symplectic geometry in Hilbert space and the spectral theory of nonselfadjoint operators. These techniques were motivated by the investigation of soliton asymtotics for integrable equations (a survey can be found in [7] and [8]), and by the methods introduced by Soffer and Weinstein [24, 25, 30]. Similar techniques were developed by Miller, Pego and Weinstein for the 1D modified KdV and RLW equations, [16, 18, 19]. The methods and results were extended in [4, 5] to one-dimensional Schrödinger equations with more complicated spectral properties. In higher dimensions close results were obtained in $[6,12,22,26,27,29]$ for nonlinear Schrödinger equations, and in [10] for three-dimensional translation invariant wave-particle system.

Let us comment on the general strategy of our proofs. We develop the approach $[3,10]$ for our problem. Firstly, we apply the symplectic projection onto the solitary manifold to separate the motion along the solitary manifold and in transversal direction. Secondly, we derive the modulation equations for the parameters of the symplectic projection, and linearize the transversal dynamics at the projection of the trajectory. The linearized equation is nonautonomous, and this is one of the fundamental difficulties in the proof. This difficulty is handled by the introduction of an autonomous equation (by freezing the time) with an application of the modulation equations to estimate the resulting additional error terms. A principal role in the rest of the proof is played by the uniform decay of the frozen linearized dynamics projected onto the continuous spectrum, and the method of majorants.

Let us note the following two main novelties in our approach to the uniform decay. First, we calculate exactly all needed spectral properties of corresponding generator. Second, we do not use a spectral representation of the generator. Instead, we develop the Jensen-Kato approach applying directly the Zygmund type Lemma 6.1 (cf. [11, Lemma 10.2]) to the Laplace integral of the resolvent. We expect that the development would be promising for more general problems.

The paper is organized as follows. In Section 2 some notation and definitions are given. In Section 3 we describe all nonzero solitary waves and formulate the main theorem. The linearization at a solitary wave is carried out in Section 4. In Sections 5 and 6, we construct the spectral representation for the linearized equation. In Section 7 we establish the time decay for the linearized equation in the continuous spectrum. In Section 9 the modulation equations for the parameters of the soliton are displayed. The decay of the transverse component is proved in Sections 10,11. In Section 12 we obtain the soliton asymptotics (1.2). In Appendix we study the resolvent of linearized equation.

In conclusion, we expect that the asymptotics (1.2) holds for any finite energy solution of the equation (1.1), however this is still open problem. We hope to prove it combining our methods with the techniques of the papers $[14,15]$, where global attraction to the solitary manifold is proved for the 1D Klein-Gordon equation with the same delta-nonlinearity. We also intend to treat in a later publication the case when the linearization has nontrivial stable oscillatory modes (which occurs if (3.2) holds but (3.3) does not).

\section{Notation and definitions}

We identify a complex number $\psi=\psi_{1}+i \psi_{2}$ with the real two-dimensional vector $\left(\psi_{1}, \psi_{2}\right) \in \mathbb{R}^{2}$ and assume that the vector version $\mathbf{F}$ of the oscillator force $F$ admits a real-valued potential,

$$
\mathbf{F}(\psi)=-\nabla U(\psi), \quad \psi \in \mathbb{R}^{2}, \quad U \in C^{2}\left(\mathbb{R}^{2}\right) .
$$


Then (1.1) is formally a Hamiltonian system with Hamiltonian

$$
\mathcal{H}(\psi)=\frac{1}{2} \int\left|\psi^{\prime}\right|^{2} d x+U(\psi(0))
$$

which is conserved for sufficiently regular finite energy solutions. We assume that the potential $U(\psi)$ satisfies the inequality

$$
U(z) \geq A-B|z|^{2} \quad \text { with some } \quad A \in \mathbb{R}, \quad B>0 .
$$

Our key assumption concerns the $U(1)$-invariance of the oscillator (cf [1]), where $U(1)$ stands for the group $e^{i \theta}, \theta \in[0,2 \pi]$ acting by phase rotation $\psi \mapsto e^{i \theta} \psi$. Namely, we assume that $U(\psi)=u\left(|\psi|^{2}\right)$ with $u \in C^{2}(\mathbb{R})$. Therefore, by $(2.1)$,

$$
F(\psi)=a\left(|\psi|^{2}\right) \psi, \quad \psi \in \mathbb{C}, \quad a \in C^{1}(\mathbb{R}),
$$

where $a\left(|\psi|^{2}\right)$ is real. Then $F\left(e^{i \theta} \psi\right)=e^{i \theta} F(\psi), \quad \theta \in[0,2 \pi]$ and $F(0)=0$ for continuous $F$. Obviously, the symmetry holds true if $U(\psi)=u\left(|\psi|^{2}\right)$. The symmetry implies that $e^{i \theta} \psi(x, t)$ is a solution to (1.1) if $\psi(x, t)$ is. The equation is $U(1)$-invariant in the sense of [9], and the Nöther theorem implies the charge conservation:

$$
\mathcal{Q}(\psi)=\int|\psi|^{2} d x=\text { const } .
$$

The main subject of this paper is an analysis of the special role played by "quantum stationary states", or solitary waves in the sense of [9], which are finite energy solutions of the form

$$
\psi(x, t)=\psi_{\omega}(x) e^{i \omega t}, \quad \omega \in \mathbb{R} .
$$

The frequency $\omega$ and the amplitude $\psi_{\omega}(x)$ solve the following nonlinear eigenvalue problem:

$$
-\omega \psi_{\omega}(x)=-\psi_{\omega}^{\prime \prime}(x)-\delta(x) F\left(\psi_{\omega}(0)\right), \quad x \in \mathbb{R} .
$$

which follows directly from (1.1) and (2.4) since $\omega \in \mathbb{R}$.

Definition 2.1 $\mathcal{S}$ denotes the set of all nonzero solutions $\psi_{\omega}(x) \in H^{1}(\mathbb{R})$ to (2.7) with all possible $\omega \in \mathbb{R}$.

Here $H^{1}(\mathbb{R})=H^{1}$ denotes the Sobolev space of complex valued measurable functions with $\int\left(\left|\psi^{\prime}\right|^{2}+|\psi|^{2}\right) d x<\infty$. We give below in section 3 a complete analysis of the set $\mathcal{S}$ of all nonzero solitary waves $\psi_{\omega}(x)$ by an explicit calculation: it consists of functions $C(\omega) e^{-\sqrt{\omega}|x|+i \theta}$ with $C>0, \omega=\omega(C)>0$ and any $\theta \in[0,2 \pi]$, and $C$ restricted to lie in a set which, in the case of polynomial $F$, is a finite union of one-dimensional intervals. Notice that $C=0$ corresponds to the zero function $\psi(x)=0$ which is always a solitary wave as $F(0)=0$, and for $\omega \leq 0$ only the zero solitary wave exists.

Our main results describe the large time behavior of the global solutions whose existence is guaranteed by the following theorem, which is proved in [13].

Theorem 2.2 i) Let conditions (2.1), (2.3) and (2.4) hold. Then for any $\psi_{0}(x) \in H^{1}$ there exist a unique solution $\psi(x, t) \in C_{b}\left(\mathbb{R}, H^{1}\right)$ to the equation (1.1) with initial condition $\psi(x, 0)=$ $\psi_{0}(x)$.

ii) There exists $\Lambda\left(\psi_{0}\right)>0$ such that the following a priori bound holds:

$$
\sup _{t \in \mathbb{R}}\|\psi(t)\|_{H^{1}} \leq \Lambda\left(\psi_{0}\right)<\infty .
$$


The functional spaces we are going to consider are the weighted Banach spaces $L_{\beta}^{p}, p \in$ $[1, \infty), \beta \in \mathbb{R}$ of complex valued measurable functions with the norm

$$
\|u\|_{L_{\beta}^{p}}=\left\|(1+|x|)^{\beta} u(x)\right\|_{L^{p}} .
$$

\section{Solitary waves and statement of the main theorem}

Lemma 3.1 The set of all nonzero solitary waves is given by

$$
\mathcal{S}=\left\{\psi_{\omega} e^{i \theta}=C e^{i \theta-\sqrt{\omega}|x|}: \omega>0, \quad C>0, \quad \sqrt{\omega}=a\left(C^{2}\right) / 2>0, \quad \theta \in[0,2 \pi]\right\} .
$$

Proof Let us calculate all solitary waves (2.6). The equation (2.7) implies $\psi^{\prime \prime}(x)=\omega \psi(x)$ for $x \neq 0$, hence

$$
\psi(x)=C e^{-\kappa|x|}, \quad x \in \mathbb{R}
$$

where $\kappa=\sqrt{\omega}>0$ and $\omega>0$ since $\psi(x)$ is continuous, and $\psi(x) \in L^{2}$. Equating the coefficients of $\delta(x)$ in both sides of $(2.7)$, we get:

$$
0=\psi^{\prime}(0+)-\psi^{\prime}(0-)+F(\psi(0)) .
$$

This implies $0=-2 \kappa C+F(C)$, or equivalently,

$$
\kappa=\frac{F(C)}{2 C}=\frac{a\left(C^{2}\right)}{2} .
$$

Corollary 3.2 The set $\mathcal{S}$ is a smooth manifold with co-ordinates $\theta \in \mathbb{R} \bmod 2 \pi$ and $C>0$ such that $a\left(C^{2}\right)>0$.

Remark 3.3 We will analyse only the solitary waves with $a^{\prime}(C) \neq 0$. On the manifold $\mathcal{S}$ we have $\omega=\kappa^{2}$ with $\kappa=a\left(C^{2}\right) / 2$ according to (3.1). Hence, the parameters $\theta, \omega$ locally also are smooth coordinates on $\mathcal{S}$ at the points with $a^{\prime}=a^{\prime}(C) \neq 0$ since $\omega^{\prime}=2 \kappa \kappa^{\prime}=a a^{\prime} C \neq 0$ then.

The soliton solution is a trajectory $\psi_{\omega(t)}(x) e^{i \theta(t)}=C e^{-\sqrt{\omega(t)}|x|} e^{i \theta(t)}$, where the parameters satisfy the equation $\dot{\theta}=\omega, \dot{\omega}=0$. The solitary waves $e^{i \theta} \psi_{\omega}(x)$ map out in time an orbit of the $U(1)$ symmetry group. This group acts on the phase space $H^{1}(\mathbf{R})$ preserving $\mathcal{H}$ and the standard symplectic form (6.3); in other words the solitary waves (2.6) are relative equilibria of the corresponding Hamiltonian system.

Let us denote $N(C)=\int\left|\psi_{\omega}(x)\right|^{2} d x$ with $\omega=\kappa^{2}$, and $\kappa=a\left(C^{2}\right) / 2$ according to (3.1). It is easy to compute that $N(C)=C^{2} / \kappa$. We now differentiate:

$$
N^{\prime}(C)=\frac{2 C}{\kappa}-\frac{C^{2} \kappa^{\prime}}{\kappa^{2}} .
$$

Differentiating the identity (3.1), we obtain $\kappa^{\prime}=a^{\prime} C$. Thus, again by (3.1),

$$
N^{\prime}(C)=\frac{2 C}{\kappa}\left(1-\frac{a^{\prime} C^{2}}{a}\right) \neq 0
$$

if $C>0, a>0$ and $a^{\prime} \neq a / C^{2}$. Therefore noticing that $N^{\prime}(C)=\omega^{\prime}(C) \partial_{\omega} \int\left|\psi_{\omega}\right|^{2} d x$ with $\omega^{\prime}(C)=2 \kappa \kappa^{\prime}=a a^{\prime} C$, we obtain the following result 
Lemma 3.4 For $C>0, a>0$ we have

$$
\partial_{\omega} \int\left|\psi_{\omega}(x)\right|^{2} d x<0 \quad \text { if } a^{\prime} \in(-\infty, 0) \cup\left(a / C^{2},+\infty\right),
$$

and

$$
\partial_{\omega} \int\left|\psi_{\omega}(x)\right|^{2} d x>0 \text { if } 0<a^{\prime}<a / C^{2}
$$

Remark 3.5 (i) Orbital stability of solitary waves is a much studied subject (see [9] for very general theorems in this area, and [28] for an approach more similar to that taken in this paper). The standard condition for orbital stability $([9])$ for the present problem would read $\partial_{\omega} \int\left|\psi_{\omega}(x)\right|^{2} d x>0$; this is expected to be a necessary and sufficient condition for orbital stability when the Hessian of the augmented Hamiltonian ([28]) has a single negative eigenvalue. In the present problem it can be easily calculated that this Hessian is non-negative when $a^{\prime}<0$ and thus the standard condition is not necessarily relevant if $a^{\prime}<0$. Indeed Theorem 3.7 asserts stability in the case $a^{\prime}<0$. Restricting to $a^{\prime}>0$, in which case the Hessian does have a single negative eigenvalue, the calculation above shows that orbital stability is expected to hold when $a^{\prime}<a / C^{2}$. In this paper we will work under the spectral condition (3.3) which, for $a^{\prime}>0$, is slightly stricter: it is imposed to ensure that the linearization has no discrete spectrum except zero (which is always present on account of the circular symmetry of the problem). If $a / \sqrt{2} C^{2}<a^{\prime}<a / C^{2}$ there are two purely imaginary eigenvalues of the linearized operator. It is intended to treat this case in a later publication thus extending our proof of asymptotic stability to the entire range

$$
-\infty<a^{\prime}<a / C^{2}
$$

For $a^{\prime}>a / C^{2}$ the linearized operator has a positive eigenvalue and the solitary wave is linearly unstable.

(ii) It is explained at the end of section 4 that (3.4) can be interpreted as saying the restriction of the standard symplectic form (6.3) to the tangent space to $\mathcal{S}$ is non-degenerate (i.e. $\mathcal{S}$ satisfies the condition to be a symplectic submanifold).

Definition 3.6 We say the solitary wave $\psi_{\omega}(x) e^{i \theta}=C e^{-\sqrt{\omega}|x|+i \theta}, C>0$ satisfies the spectral condition if $\omega>0$ and (cf. Remark 3.3)

$$
a^{\prime}\left(C^{2}\right) \in(-\infty, 0) \cup\left(0, a\left(C^{2}\right) /\left(\sqrt{2} C^{2}\right)\right) .
$$

Let us denote by $W(t)$ the dynamical group of the free Schrödinger equation: $W(t) f$ is defined by the Fourier representation for all tempered distributions $f$. Our main theorem is the following:

Theorem 3.7 Let conditions (2.1), (2.3) and (2.4) hold, $\beta \geq 2$ and $\psi(x, t) \in C\left(\mathbb{R}, H^{1}\right)$ be the solution to the equation (1.1) with initial value $\psi_{0}(x)=\psi(x, 0) \in H^{1} \cap L_{\beta}^{1}$ which is close to a solitary wave $\psi_{\omega_{0}} e^{i \theta_{0}}=C_{0} e^{-\sqrt{\omega_{0}}|x|+i \theta_{0}}$ with $C_{0}>0$ and $\omega_{0}>0$ :

$$
d:=\left\|\psi_{0}-\psi_{\omega_{0}} e^{i \theta_{0}}\right\|_{H^{1} \cap L_{\beta}^{1}} \ll 1 .
$$

Assume further that the spectral condition (3.3) holds for the solitary wave with $C=C_{0}$. Then for d sufficiently small the solution admits the following asymptotics:

$$
\psi(\cdot, t)=\psi_{\omega_{ \pm}} e^{i \omega_{ \pm} t}+W(t) \Phi_{ \pm}+r_{ \pm}(t), \quad t \rightarrow \pm \infty,
$$

where $\Phi_{ \pm} \in C_{b}(\mathbb{R}) \cap L^{2}(\mathbb{R})$ are the corresponding asymptotic scattering states, and

$$
\left\|r_{ \pm}(t)\right\|_{C_{b}(\mathbb{R}) \cap L^{2}(\mathbb{R})}=\mathcal{O}\left(|t|^{-1 / 2}\right), \quad t \rightarrow \pm \infty .
$$


Remark 3.8 It is possible to derive further information about the structure of $\Phi_{ \pm}$and $r_{ \pm}(t)$ as discussed towards the end of section 10.

\section{Linearization on the solitary wave}

As the first step in the proof of main theorem, let us linearize the nonlinear Schrödinger equation (1.1) at a solitary wave $e^{i(\omega t+\theta)} \psi_{\omega}(x)$, with $\psi_{\omega}(x)=C e^{-\kappa|x|}$ where $\kappa=\sqrt{\omega}>0$ and $C>0$. Substituting

$$
\psi(x, t)=e^{i(\omega t+\theta)}\left(\psi_{\omega}(x)+\chi(x, t)\right)
$$

to (1.1), we obtain by $(2.7)$ that

$$
i \dot{\chi}(x, t)=-\chi^{\prime \prime}(x, t)+\omega \chi(x, t)-\delta(x)\left[a\left(C^{2}\right) \chi(0, t)+a^{\prime}\left(C^{2}\right) C^{2} 2 \operatorname{Re} \chi(0, t)\right]+\mathcal{O}\left(|\chi|^{2}\right) .
$$

It is evident that the first order part is not linear over the complex field. On the other hand, it is linear over the real field. Hence, it would be useful to rewrite the first order part of (4.2) in the real form. Namely, identify $\chi=\chi_{1}+i \chi_{2} \in \mathbb{C}$ with the real vector $\left(\chi_{1}, \chi_{2}\right) \in \mathbb{R}^{2}$ and denote it again by $\chi$. Then the first order part of (4.2) becomes

$$
j \dot{\chi}(x, t)=-\chi^{\prime \prime}(x, t)+\omega \chi(x, t)-\delta(x)\left[a\left(C^{2}\right)+2 a^{\prime}\left(C^{2}\right) C^{2} P_{1}\right] \chi(0, t),
$$

where $P_{1}$ is the projector in $\mathbb{R}^{2}$ acting as $\left(\begin{array}{l}\chi_{1} \\ \chi_{2}\end{array}\right) \mapsto\left(\begin{array}{l}\chi_{1} \\ 0\end{array}\right)$, and $j=\left(\begin{array}{cc}0 & -1 \\ 1 & 0\end{array}\right)$. Respectively, we also rewrite (1.1) in the real form

$$
j \dot{\psi}(x, t)=-\psi^{\prime \prime}(x, t)-\delta(x) \mathbf{F}(\psi(0, t)),
$$

as an equation for $\psi(x, t) \in \mathbb{R}^{2}$ with $\mathbf{F}(\psi) \in \mathbb{R}^{2}$ which is the real vector version of $F(\psi) \in \mathbb{C}$. Then the linearization (4.3) reads as

$$
j \dot{\chi}(x, t)=-\chi^{\prime \prime}(x, t)+\omega \chi(x, t)-\delta(x) \mathbf{F}^{\prime}((C, 0)) \chi(0, t) .
$$

Here $\mathbf{F}^{\prime}$ is the differential of the map $\mathbf{F}: \mathbb{R}^{2} \rightarrow \mathbb{R}^{2}$,

$$
\mathbf{F}^{\prime}((C, 0))=a+b P_{1}, \quad a:=a\left(C^{2}\right), \quad b:=2 a^{\prime}\left(C^{2}\right) C^{2} .
$$

In order to apply the Laplace transform the next step is to complexify the system (4.5) i.e. to consider it as a system of equations for the complex functions $\chi_{1}(x, t), \chi_{2}(x, t)$, so $\chi(x, t) \in \mathbb{C}^{2}$ for any fixed $(x, t)$. This gives a system which is linear over the complex field allowing application of the Laplace transform. To write this system more concisely let us denote the complex linear operator

$$
\mathbf{B}=-\frac{d^{2}}{d x^{2}}+\omega-\delta(x) \mathbf{F}^{\prime}((C, 0))=\left(\begin{array}{cc}
\mathbf{D}_{1} & 0 \\
0 & \mathbf{D}_{2}
\end{array}\right)
$$

where

$$
\mathbf{D}_{1}=-\frac{d^{2}}{d x^{2}}+\omega-\delta(x)[a+b], \quad \mathbf{D}_{2}=-\frac{d^{2}}{d x^{2}}+\omega-\delta(x) a .
$$

The system (4.5) then reads as

$$
\dot{\chi}(x, t)=\mathbf{C} \chi(x, t), \quad \mathbf{C}:=j^{-1} \mathbf{B}=\left(\begin{array}{rr}
0 & \mathbf{D}_{2} \\
-\mathbf{D}_{1} & 0
\end{array}\right) .
$$

Theorem 2.2 generalises to the equation (4.9): the equation admits unique solution $\chi(x, t) \in$ $C_{b}\left(\mathbb{R}, H^{1}\right)$ for every initial function $\chi(x, 0)=\chi_{0} \in H^{1}$. Denote by $e^{\mathbf{C} t}$ the dynamical group of equation (4.9) acting in the space $H^{1}$. 


\section{Laplace transform}

Equation (4.9) can be solved by the Laplace transform $\tilde{\chi}(x, \omega):=\int_{0}^{\infty} e^{-\lambda t} \chi(x, t) d t$. The Laplace transform is analytic function in the complex halfplane $\operatorname{Re} \lambda>0$ with the values in $H^{1}$ since the solution is bounded in $H^{1}$. This implies that the resolvent $\mathbf{R}(\lambda):=(\mathbf{C}-\lambda)^{-1}$ is also analytic for $\operatorname{Re} \lambda>0$, with values in the space of bounded operators on $H^{1}$. From the inversion of the Laplace transform we obtain

$$
e^{\mathbf{C} t}=-\frac{1}{2 \pi i} \int_{-i \infty}^{i \infty} e^{\lambda t} \mathbf{R}(\lambda+\varepsilon) d \lambda, \quad t>0
$$

for any $\varepsilon>0$, where the integral converges in the sense of distributions of $t \in \mathbb{R}$.

We assume that the spectral condition (3.3) holds from now on. Then the resolvent admits analytic continuation from $\operatorname{Re} \lambda>0$ to the complex plain with the cuts $\mathcal{C}_{+}=[i \omega, i \infty), \mathcal{C}_{-}=$ $(-i \infty,-i \omega]$, and with the pole of order two at $\lambda=0$ as detailed in Appendix A. Furthermore, for $\lambda \in \mathcal{C}_{+} \cup \mathcal{C}_{-}$, the resolvent $\mathbf{R}(\lambda \pm \varepsilon)$ has right and left limits $\mathbf{R}(\lambda \pm 0)$ as $\varepsilon \rightarrow 0$. Then (5.1) implies that

$$
e^{\mathbf{C} t}=-\frac{1}{2 \pi i} \int_{|\lambda|=r} e^{\lambda t} \mathbf{R}(\lambda) d \lambda-\frac{1}{2 \pi i} \int_{\mathcal{C}_{+} \cup \mathcal{C}_{-}} e^{\lambda t}(\mathbf{R}(\lambda+0)-\mathbf{R}(\lambda-0)) d \lambda, \quad \text { for any } \quad r \in(0, \omega)
$$

by the Cauchy theorem. Setting $t=0$, we obtain that

$$
1=-\frac{1}{2 \pi i} \int_{|\lambda|=r} \mathbf{R}(\lambda) d \lambda-\frac{1}{2 \pi i} \int_{\mathcal{C}_{+} \cup \mathcal{C}_{-}}(\mathbf{R}(\lambda+0)-\mathbf{R}(\lambda-0)) d \lambda=\mathbf{P}^{0}+\mathbf{P}^{c},
$$

where $\mathbf{P}^{0}$ and $\mathbf{P}^{c}$ stands for the corresponding Riesz projectors (see [21]) onto, respectively, the generalised null space of $\mathbf{C}$, and onto the continuous spectral subspace. We will show in the next section that $\mathbf{P}^{0}$ is the symplectic projection, and therefore, $\mathbf{P}^{c}$ is also the symplectic projection. The projectors $\mathbf{P}^{0}, \mathbf{P}^{c}$ commute with $\mathbf{C}$ and with the group $e^{\mathbf{C} t}$. Let us note that

$$
\mathbf{P}^{0} e^{\mathbf{C} t}=-\frac{1}{2 \pi i} \int_{|\lambda|=r} e^{\lambda t} \mathbf{R}(\lambda) d \lambda, \quad \mathbf{P}^{c} e^{\mathbf{C} t}=-\frac{1}{2 \pi i} \int_{\mathcal{C}_{+} \cup \mathcal{C}_{-}} e^{\lambda t}(\mathbf{R}(\lambda+0)-\mathbf{R}(\lambda-0)) d \lambda .
$$

The first equation holds since both sides are one-parameter groups of operators, and their derivatives at $t=0$ coincide. The second equation follows from (5.2) and the fact that $1=$ $\mathbf{P}^{0}+\mathbf{P}^{c}$ by (5.3). Therefore, (5.2) becomes

$$
e^{\mathbf{C} t}=\mathbf{P}^{0} e^{\mathbf{C} t}+\mathbf{P}^{c} e^{\mathbf{C} t}
$$

\section{Invariant subspace of discrete spectrum}

Here we prove that $\mathbf{P}^{0}$ is the symplectic projection onto the tangent space of the solitary manifold $\mathcal{S}$ at the solitary wave $e^{j \theta} \psi_{\omega}$. The real form of the solitary wave is $e^{j \theta} \Phi_{\omega}$ where $\Phi_{\omega}=\left(\psi_{\omega}(x), 0\right)$. The tangent space to $\mathcal{S}$ at the point $e^{j \theta} \Phi_{\omega}$ with parameters $\omega, \theta$ is the linear span of the derivatives with respect to $\theta$ and $\omega$ (cf. Remark 3.3) i.e.

$$
T_{\omega, \theta} \mathcal{S} \equiv \text { linear } \operatorname{span}\left\{j e^{j \theta} \Phi_{\omega}(x), e^{j \theta} \partial_{\omega} \Phi_{\omega}(x)\right\} .
$$


Notice that the operator $\mathbf{C}$ corresponds to $\theta=0$ since we have extracted the phase factors $e^{i \theta}$ from the solution in the process of linearization (4.1). The tangent space to $\mathcal{S}$ at the point $\Phi_{\omega}$ with parameters $(\omega, 0)$ is spanned by the vectors

$$
T_{0}(\omega):=j \Phi_{\omega}, \quad T_{1}(\omega):=\partial_{\omega} \Phi_{\omega}
$$

Observe that (2.7) and its derivative in $\omega$ give the following identities:

$$
\mathbf{D}_{2} \psi_{\omega}=0 \quad \mathbf{D}_{1}\left(\partial_{\omega} \psi_{\omega}\right)=-\psi_{\omega} .
$$

These formulae imply that the vectors $T_{0}$ and $T_{1}$ lie in the generalised null space of the nonself-adjoint operator $\mathbf{C}$ defined in (4.9) and in fact Theorem A.6 ii) implies:

Lemma 6.1 Let the spectral condition (3.3) hold. Then the generalised null space of $\mathbf{C}$ is two dimensional, is spanned by $T_{0}, T_{1}$, and

$$
\mathrm{C} T_{0}=0 \quad \mathrm{C} T_{1}=T_{0} .
$$

We also introduce the symplectic form $\Omega$ for the real vectors $\psi$ and $\eta$ by the integral

$$
\Omega(\psi, \eta)=\int\langle j \psi, \eta\rangle d x=\int\left(\psi_{1} \eta_{2}-\psi_{2} \eta_{1}\right) d x,
$$

where $\langle\cdot, \cdot$,$\rangle stands for the scalar product in \mathbb{R}^{2}$. Since $a^{\prime} \neq a / C^{2}$ then by Lemma 3.4

$$
\boldsymbol{\mu}_{\boldsymbol{\omega}}=-\Omega\left(T_{0}, T_{1}\right)=\frac{1}{2} \partial_{\omega} \int\left|\psi_{\omega}\right|^{2} d x \neq 0
$$

Hence, the symplectic form $\Omega$ is nondegenerate on the tangent space $T_{\omega, 0} \mathcal{S}$, i.e. $T_{\omega, 0} \mathcal{S}$ is a symplectic subspace. Therefore, there exists a symplectic projection operator from $L^{2}(\mathbb{R})$ onto $T_{\omega, 0} \mathcal{S}$.

Lemma 6.2 The operator $\mathbf{P}^{0}$, defined in (5.3), is precisely the symplectic projector from $L^{2}(\mathbb{R})$ onto $T_{\omega, 0} \mathcal{S}$, and, furthermore, it may be represented by the formula

$$
\mathbf{P}^{0} \psi=b_{0} T_{0}+b_{1} T_{1} \quad \text { with } \quad-\boldsymbol{\mu}_{\boldsymbol{\omega}} b_{0}=\Omega\left(\psi, T_{1}\right), \quad \boldsymbol{\mu}_{\boldsymbol{\omega}} b_{1}=\Omega\left(\psi, T_{0}\right) .
$$

Proof The coincidence of both definition (5.3) and (6.5) of operator $\mathbf{P}^{0}$ follows by the Cauchy residue theorem from the formulas (7.4)-(7.6) for the resolvent.

Corollary 6.3 $\mathbf{P}^{c}=1-\mathbf{P}^{0}$ is also symplectic projector.

Remark 6.4 Since $T_{0}(\omega), T_{1}(\omega)$ lie in $H^{1}(\mathbb{R})$ the operator $\mathbf{P}^{0}$ extends uniquely to define a continuous linear map $H^{-1}(\mathbb{R}) \rightarrow T_{\omega, 0} \mathcal{S}$, which is still designated $\mathbf{P}^{0}$. In particular this operator can be applied to the Dirac measure $\delta(x)$.

Using the Taylor expansion for the $e^{\lambda t}$ at $\lambda=0$ and the identity $\lambda \mathbf{R}(\lambda)=\mathbf{C R}(\lambda)-1$, we obtain by (5.4)

$$
\mathbf{P}^{0} e^{\mathbf{C} t}=(1+\mathbf{C} t) \mathbf{P}^{0}
$$

Remark 6.5 On the generalised null space itself $\mathbf{C}^{2}=0$ by Lemma 6.1 and so the semigroup $e^{t \mathbf{C}}$ reduces to $1+\mathbf{C} t$ as usual for the exponential of the nilpotent part of an operator. 


\section{Time decay in continuous spectrum}

From formulas $\left(5.5,(6.6)\right.$ we see that the solutions $\chi(t)=e^{\mathbf{C} t} \chi_{0}$ of the linearized equation (4.9) do not decay as $t \rightarrow \infty$ if $\mathbf{P}^{0} \chi_{0} \neq 0$. On the other hand, we do expect time decay of $\mathbf{P}^{c} \chi(t)$, as a consequence of the Laplace representation (5.4) for $\mathbf{P}^{c} e^{\mathbf{C} t}$ :

$$
\mathbf{P}^{c} e^{\mathbf{C} t}=-\frac{1}{2 \pi i} \int_{\mathcal{C}_{+} \cup \mathcal{C}_{-}} e^{\lambda t}(\mathbf{R}(\lambda+0)-\mathbf{R}(\lambda-0)) d \lambda .
$$

For $\beta \geq 2$ let us introduce a Banach space $\mathcal{M}_{\beta}$, which is the subset of distributions which are linear combinations of $L_{\beta}^{1}$ functions and multiples of the Dirac distribution at the origin with the norm:

$$
\|\psi+C \delta(x)\|_{\mathcal{M}_{\beta}}:=\|\psi\|_{L_{\beta}^{1}}+|C| .
$$

and let $\mathcal{B}=B\left(\mathcal{M}_{\beta}, L_{-\beta}^{\infty}\right)$ be a Banach space of continuous linear maps $\mathcal{M}_{\beta} \rightarrow L_{-\beta}^{\infty}$.

Theorem 7.1 Assume that the spectral condition (3.3) holds. Then for $\beta \geq 2$

$$
\left\|\mathbf{P}^{c} e^{\mathbf{C} t}\right\|_{\mathcal{B}}=\mathcal{O}\left(t^{-3 / 2}\right), \quad t \rightarrow \infty
$$

\section{Proof}

Step i) The resolvent $\mathbf{R}(\lambda)$ is an integral operator with matrix valued integral kernel

$$
\mathbf{R}(\lambda, x, y)=\Gamma(\lambda, x, y)+P(\lambda, x, y)
$$

where

$$
\begin{gathered}
\Gamma(\lambda, x, y)=\left(\begin{array}{cc}
\frac{1}{4 k_{+}} & -\frac{1}{4 k_{-}} \\
\frac{i}{4 k_{+}} & \frac{i}{4 k_{-}}
\end{array}\right)\left(\begin{array}{cc}
e^{i k_{+}|x-y|}-e^{i k_{+}(|x|+|y|)} & -i\left(e^{i k_{+}|x-y|}-e^{i k_{+}(|x|+|y|)}\right) \\
e^{i k_{-}|x-y|}-e^{i k_{-}(|x|+|y|)} & i\left(e^{i k_{-}|x-y|}-e^{i k_{-}(|x|+|y|)}\right)
\end{array}\right) \\
P(\lambda, x, y)=\frac{1}{2 D}\left(\begin{array}{cc}
e^{i k_{+}|x|} & e^{i k_{-}|x|} \\
i e^{i k_{+}|x|} & -i e^{i k_{-}|x|}
\end{array}\right)\left(\begin{array}{cc}
i \alpha-2 k_{-} & i \beta \\
-i \beta & -i \alpha+2 k_{+}
\end{array}\right)\left(\begin{array}{cc}
e^{i k_{+}|y|} & -i e^{i k_{+}|y|} \\
e^{i k_{-}|y|} & i e^{i k_{-}|y|}
\end{array}\right) .
\end{gathered}
$$

Here $k_{ \pm}(\lambda)=\sqrt{-\omega \mp i \lambda}$ is the square root defined with cuts in the complex $\lambda$ plane so that $k_{ \pm}(\lambda)$ is analytic on $\mathbb{C} \backslash \mathcal{C}_{ \pm}$and $\operatorname{Im} k_{ \pm}(\lambda)>0$ for $\lambda \in \mathbb{C} \backslash \mathcal{C}_{ \pm}$. The constants $\alpha, \beta$ and $D=D(\lambda)$ are given by the formulas

$$
\alpha=a+b / 2, \beta=b / 2, D=2 i \alpha\left(k_{+}+k_{-}\right)-4 k_{+} k_{-}+\alpha^{2}-\beta^{2} .
$$

We prove these formulas in Appendix A. Also in Appendix A we prove that $D(\lambda) \neq 0$ for $\lambda \in \mathcal{C}_{+} \cup \mathcal{C}_{-}$. Formulas (7.1) and (7.4) imply

$$
-2 \pi i \mathbf{P}^{c} e^{\mathbf{C} t}=\int_{\mathcal{C}_{+} \cup \mathcal{C}_{-}} e^{\lambda t}(\Gamma(\lambda+0)-\Gamma(\lambda-0)) d \lambda+\int_{\mathcal{C}_{+} \cup \mathcal{C}_{-}} e^{\lambda t}(P(\lambda+0)-P(\lambda-0)) d \lambda
$$

Step ii) Clearly in order to understand the decay of $\mathbf{P}^{c} e^{t \mathbf{C}}$, it is crucial to study the behaviour of $\Gamma(\lambda+0)-\Gamma(\lambda-0)$ and $P(\lambda+0)-P(\lambda-0)$ near the branch points $\lambda= \pm i \omega$ (where $k_{ \pm}$ vanish).

We deduce time decay for the group $\mathbf{P}^{c} e^{t \mathbf{C}}$ by means of the following version of Lemma 10.2 from [11], which is itself based on Zygmund's lemma [31, p.45]. 
Lemma 7.2 Let $\mathcal{F}:[0, \infty) \rightarrow \mathbf{B}$ be a $C^{2}$ function with values in a Banach space $\mathbf{B}$. Suppose that $\mathcal{F}(0)=0, \mathcal{F}^{\prime \prime} \in L^{1}(\delta, \infty ; \mathbf{B})$ for some $\delta>0$ and

$$
\mathcal{F}^{\prime \prime}(\zeta)=\mathcal{O}\left(\zeta^{p-2}\right), \quad \zeta \rightarrow 0
$$

in the norm of $\mathbf{B}$ for some $p \in(0,1)$. Then for any $\varepsilon>0$

$$
I(t)=\int_{0}^{\infty} e^{-i t \zeta} \mathcal{F}(\zeta) d \zeta \in C_{b}(\varepsilon, \infty ; \mathbf{B})
$$

and

$$
I(t)=\mathcal{O}\left(t^{-1-p}\right) \quad \text { as } \quad t \rightarrow \infty
$$

in the norm of $\mathbf{B}$.

Next we apply Lemma 7.2 to each summand in the RHS of (7.7) separately.

Step iii) Let us consider the first summand in the RHS of (7.7). We consider only the integral over $\mathcal{C}_{+}$since the integral over $\mathcal{C}_{-}$can be handled in the same way. The point $\lambda=i \omega$ is the branch point for $k_{+}$, therefore, if $\lambda \in \mathcal{C}_{+}$then since $k_{-}$is continuous across $\mathcal{C}_{+}$

$$
\Gamma(\lambda+0)-\Gamma(\lambda-0)=\Gamma^{+}(\lambda+0)-\Gamma^{+}(\lambda-0)
$$

where $\Gamma^{+}$is the sum of those terms in $\Gamma$ which involve $k_{+}$. Let us consider, for example, $\Gamma_{11}^{+}$. The expression (7.5) implies for $y>0$ that

$$
\Gamma_{11}^{+}(\lambda, x, y)= \begin{cases}0, & x \leq 0 \\ \frac{e^{i k_{+} y}\left(e^{-i k_{+} x}-e^{i k_{+} x}\right)}{4 k_{+}}, & 0 \leq x \leq y \\ \frac{e^{i k_{+} x}\left(e^{-i k_{+} y}-e^{i k_{+} y}\right)}{4 k_{+}}, & x \geq y .\end{cases}
$$

For $\lambda \in \mathcal{C}_{+}$, the root $k_{+}=\sqrt{-\omega-i \lambda}$ is real, and $k_{+}(\lambda+0)=-k_{+}(\lambda-0)$. Then, for $y>0$,

$$
\Gamma_{11}^{+}(\lambda+0, x, y)-\Gamma_{11}^{+}(\lambda-0, x, y)=-\Theta(x) \frac{\sin (\sqrt{\zeta}|x|) \sin (\sqrt{\zeta}|y|)}{\sqrt{\zeta}}
$$

where $\zeta=-\omega-i \lambda$, and $\Theta(x)=1$ for $x>0$ and zero otherwise. The second derivative of the function $f(\zeta)=\frac{\sin (\sqrt{\zeta}|x|) \sin (\sqrt{\zeta}|y|)}{\sqrt{\zeta}}$ satisfies

$$
\begin{aligned}
& \left|f^{\prime \prime}(\zeta)\right|=\mid-\frac{\sin (\sqrt{\zeta}|x|) \sin (\sqrt{\zeta}|y|)\left(|x|^{2}+|y|^{2}\right)}{4 \zeta \sqrt{\zeta}}+\frac{2 \cos (\sqrt{\zeta}|x|) \cos (\sqrt{\zeta}|y|)|x||y|}{4 \zeta \sqrt{\zeta}} \\
& -\frac{\sin (\sqrt{\zeta}|x|) \cos (\sqrt{\zeta}|y|)|y|+\cos (\sqrt{\zeta}|x|) \sin (\sqrt{\zeta}|y|)|x|}{2 \zeta^{2}} \mid \leq \frac{C\left(1+|x|^{2}\right)\left(1+|y|^{2}\right)}{\zeta \sqrt{\zeta}} .
\end{aligned}
$$


For $y<0$ an identical calculation leads to the same bound. Therefore the operator valued function $\mathcal{F}(\zeta)=\Gamma_{11}^{+}(\lambda+0)-\Gamma_{11}^{+}(\lambda-0)$ satisfies the conditions of Lemma 7.2 with $\zeta=-\omega-i \lambda$, $p=1 / 2$ and $\mathbf{B}=\mathcal{B}$. Then

$$
\int_{\mathcal{C}_{+} \cup \mathcal{C}_{-}} e^{\lambda t}(\Gamma(\lambda+0)-\Gamma(\lambda-0)) d \lambda=\mathcal{O}\left(t^{-3 / 2}\right), \quad t \rightarrow \infty
$$

in the norm $\mathcal{B}$.

Step iv) Let us consider the second summand in the RHS of (7.7). We consider only the integral over $\mathcal{C}_{+}$and one component of the matrix $P$, for example, $P_{11}$ :

$$
P_{11}(\lambda, x, y)=\frac{\left(i \alpha-2 k_{-}\right) e^{i k_{+}(|x|+|y|)}+\left(-i \alpha+2 k_{+}\right) e^{i k_{-}(|x|+|y|)}+i \beta\left(e^{i k_{-}|y|+i k_{+}|x|}-e^{i k_{+}|y|+i k_{-}|x|}\right)}{2 i \alpha\left(k_{+}+k_{-}\right)-4 k_{+} k_{-}+\alpha^{2}-\beta^{2}} .
$$

Denote $\zeta=-\omega-i \lambda$, then $k_{+}=\sqrt{\zeta}, k_{-}=\sqrt{-2 \omega-\zeta}$. A Taylor expansion in $\sqrt{\zeta}$ as $\zeta \rightarrow$ $0, \quad \operatorname{Im} \zeta \geq 0$ implies

$$
P_{11}(\lambda, x, y)=P_{0}+P_{1}(x, y) \zeta^{1 / 2}+P_{2}(x, y) \mathcal{O}(\zeta)
$$

where $\left|P_{j}(x, y)\right| \leq C_{j}\left(1+|x|^{j}\right)\left(1+|y|^{j}\right), j=1,2$. Therefore, if $\lambda \in \mathcal{C}_{+}$then

$$
\mathcal{F}(\zeta)=P_{11}(\lambda+0)-P_{11}(\lambda-0)=\mathcal{O}\left(\zeta^{1 / 2}\right), \quad \zeta \rightarrow 0
$$

in the norm of $\mathcal{B}$. Similarly, differentiating two times the function $P_{11}(\lambda, x, y)$ in $\lambda$, we obtain that

$$
\mathcal{F}^{\prime \prime}(\zeta)=-P_{11}^{\prime \prime}(\lambda+0)+P_{11}^{\prime \prime}(\lambda-0)=\mathcal{O}\left(\zeta^{-3 / 2}\right), \quad i, j=1,2, \quad \zeta \rightarrow 0
$$

in the norm of $\mathcal{B}$. Moreover, $\mathcal{F}^{\prime \prime}(\zeta) \sim \zeta^{-3 / 2}$ as $\zeta \rightarrow \infty$. Therefore, the function $\mathcal{F}(\zeta)$ satisfies the conditions of Lemma 7.2 with $p=1 / 2$. Then

$$
\int_{\mathcal{C}_{+} \cup \mathcal{C}_{-}} e^{\lambda t}(P(\lambda+0)-P(\lambda-0)) d \lambda=\mathcal{O}\left(t^{-3 / 2}\right)
$$

in the norm $\mathcal{B}$. and $\mathbf{B}=\mathcal{B}$. From (7.7), (7.10) and (7.11) the statement of theorem follows.

\section{Bounds for small $t$}

As a starting point for the method of majorants in Section 10 we will need also some estimates on the group $e^{\mathbf{C} t}$ for small $t$. First note that the function $e^{\mathbf{C} t} \chi_{0}$ belongs to $C_{b}\left(\mathbb{R}, H^{1}\right)$. This follows from a theorem analogous to Theorem 2.2 for solutions $e^{\mathrm{C} t} \chi_{0}$ of the linearized equation (4.9), with initial condition $\chi_{0} \in H^{1}$. Moreover, energy and charge conservation imply that

$$
\left\|e^{\mathbf{C} t} \chi_{0}\right\|_{H^{1}} \leq c\left\|\chi_{0}\right\|_{H^{1}}, \quad t \in \mathbb{R} .
$$

For a further application in section 11.4 we need a bound for the action of $e^{\mathbf{C} t}$ on the Dirac distribution $\delta=\delta(x)$. Thus let $\chi_{\delta}(x, t)$ be the solution to the linearized equation (4.2) with $\chi_{\delta}(x, 0)=\delta(x)$ and $e^{\mathbf{C} t} \delta$ its real vector version (with values in $\mathbb{R}^{2}$ ). Note that, by Theorem 7.1 , we have $e^{\mathbf{C} t} \delta \in C_{b}\left(\varepsilon, \infty ; L_{-\beta}^{\infty}\right)$, for every $\varepsilon>0$, and $\beta \geq 2$. The next lemma gives the small $t$ behaviour: 
Lemma 8.1 The following bound holds

$$
\left\|e^{\mathrm{C} t} \delta\right\|_{L^{\infty}}=\mathcal{O}\left(t^{-1 / 2}\right), \quad t \rightarrow 0
$$

Proof By the Duhamel representation for the solution to (4.2), we obtain

$$
\chi_{\delta}(x, t)=W_{\omega}(t) \delta-\int_{0}^{t} d s\left(a \chi_{\delta}(0, s)+b \operatorname{Re}\left(\chi_{\delta}(0, s)\right) W_{\omega}(t-s) \delta\right.
$$

where $a$ and $b$ are defined by (4.6), and $W_{\omega}(t)$ is the dynamical group of the modified Schrödinger equation

$$
i \dot{\chi}(x, t)=-\chi^{\prime \prime}(x, t)+\omega \chi(x, t) .
$$

Note that

$$
W_{\omega}(t) \delta=\frac{1}{\sqrt{4 \pi i t}} e^{i \frac{x^{2}}{4 t}-i \omega t}
$$

Therefore (8.3) becomes

$$
\chi_{\delta}(x, t)=\frac{1}{\sqrt{4 \pi i t}} e^{i \frac{x^{2}}{4 t}-i \omega t}-\int_{0}^{t} \frac{1}{\sqrt{4 \pi i(t-s)}} e^{i \frac{x^{2}}{4(t-s)}-i \omega(t-s)}\left(a \chi_{\delta}(0, s)+b \operatorname{Re}\left(\chi_{\delta}(0, s)\right) d s\right.
$$

Denote $\varsigma(x, t)=\sqrt{t} \chi_{\delta}(x, t)$. Then

$$
\varsigma(x, t)=\frac{1}{\sqrt{4 \pi i}} e^{i \frac{x^{2}}{4 t}-i \omega t}-\sqrt{t} \int_{0}^{t} \frac{1}{\sqrt{4 \pi i(t-s) s}} e^{i \frac{x^{2}}{4(t-s)}-i \omega(t-s)}(a \varsigma(0, s)+b \operatorname{Re}(\varsigma(0, s)) d s .
$$

Therefore,

$$
\|\varsigma(t)\|_{L^{\infty}} \leq \frac{1}{2 \sqrt{\pi}}+\frac{1}{2} \sqrt{\pi t}(|a|+|b|) \int_{0}^{t} \frac{1}{\pi \sqrt{(t-s) s}}\|\varsigma(s)\|_{L^{\infty}} d s, \quad t>0 .
$$

Since $\int_{0}^{t} \frac{d s}{\pi \sqrt{(t-s) s}}=1$, we obtain the bound

$$
\|\varsigma(t)\|_{L^{\infty}} \leq \frac{1}{2 \sqrt{\pi}} \frac{1}{1-\frac{1}{2} \sqrt{\pi t}(|a|+|b|)}
$$

if $t$ is sufficiently small.

\section{Modulation equations}

In this section we present the modulation equations which allow a construction of solutions $\psi(x, t)$ of the equation (1.1) close at each time $t$ to a soliton i.e. to one of the functions

$$
C e^{i \theta-\sqrt{\omega}|x|}, \quad C=C(\omega)>0
$$

in the set $\mathcal{S}$ described in section 3 with time varying ("modulating") parameters $(\omega, \theta)=$ $(\omega(t), \theta(t))$. It will be assumed that $\psi(x, t)$ is a given weak solution of (1.1) as provided by 
Theorem 2.2, so that the map $t \rightarrow \psi(\cdot, t)$ is continuous into $H^{1}(\mathbb{R})$. The modulation equations follow from the ansatz for the solution which is explained next. Recall that we defined

$$
\Phi_{\omega}(x) \equiv\left(C e^{-\sqrt{\omega}|x|}, 0\right)=\left(\psi_{\omega}, 0\right)
$$

so that $\psi(x, t)=e^{j \theta(t)} \Phi_{\omega(t)}(x)$ is a solution of (4.4) if and only if $\dot{\theta}=\omega$ and $\dot{\omega}=0$. Here it is to be understood that $C=C(\omega(t))$ is determined from $\omega(t)$ via (3.1). We look for a solution to (4.4) in the form

$$
\psi(x, t)=e^{j \theta(t)}\left(\Phi_{\omega(t)}(x)+\chi(x, t)\right)=e^{j \theta(t)} \Psi(x, t), \quad \Psi(x, t)=\Phi_{\omega(t)}(x)+\chi(x, t) .
$$

Since this is a solution of (4.4) as long as $\chi \equiv 0$ and $\dot{\theta}=\omega$ and $\dot{\omega}=0$ it is natural to look for solutions in which $\chi$ is small and

$$
\theta(t)=\int_{0}^{t} \omega(s) d s+\gamma(t)
$$

with $\gamma$ treated perturbatively. Observe that so far this representation is underdetermined since for any $(\omega(t), \theta(t))$ it just amounts to a definition of $\chi$; it is made unique by restricting $\chi(t)$ to lie in the image of the projection operator onto the continuous spectrum $\mathbf{P}_{t}^{c}=\mathbf{P}^{c}(\omega(t))$ or equivalently that

$$
\mathbf{P}_{t}^{0} \chi(t)=0, \quad \mathbf{P}_{t}^{0}=\mathbf{P}^{0}(\omega(t))=I-\mathbf{P}^{c}(\omega(t))
$$

(The projection operators are as defined in (5.3) and (6.5)). An equivalent formulation of (9.3) is to say that $e^{j \theta} \chi$ is required to lie in the symplectic normal space $N_{\omega(t), \theta(t)} \mathcal{S}$. This is equivalent to imposition of the following orthogonality conditions (at each time $t$ ):

$$
\Omega\left(\chi(t), T_{0}(\omega(t))=0=\Omega\left(\chi(t), T_{1}(\omega(t)),\right.\right.
$$

where $\Omega$ is the symplectic form introduced previously. Writing $\chi(t)=\left(\chi_{1}(t), \chi_{2}(t)\right)$ the orthogonality conditions reduce to

$$
\int \chi_{1}(x, t) C e^{-\sqrt{\omega}|x|} d x=0, \quad \text { and } \quad \int \chi_{2}(x, t) \partial_{\omega}\left(C e^{-\sqrt{\omega}|x|}\right) d x=0 .
$$

Now we give a system of modulation equations for $\omega(t), \gamma(t)$ which ensure the conditions (9.5) are preserved by the time evolution.

Lemma 9.1 (i) Assume given a solution of (4.4) with regularity as described in theorem 2.2, which can be written in the form (9.2) -(9.3) with continuously differentiable $\omega(t), \theta(t)$. Then

$$
\dot{\chi}=\mathbf{C} \chi-\dot{\omega} \partial_{\omega} \Phi_{\omega}+\dot{\gamma} j^{-1}\left(\Phi_{\omega}+\chi\right)+\mathbf{Q}
$$

where $\mathbf{Q}(\chi, \omega)=-\delta(x) j^{-1}\left(\mathbf{F}\left(\Phi_{\omega}+\chi\right)-\mathbf{F}\left(\Phi_{\omega}\right)-\mathbf{F}^{\prime}\left(\Phi_{\omega}\right) \chi\right)$, and

$$
\begin{aligned}
\dot{\omega} & =\frac{\left\langle\mathbf{P}^{0} \mathbf{Q}, \Psi\right\rangle}{\left\langle\partial_{\omega} \Phi_{\omega}-\partial_{\omega} \mathbf{P}^{0} \chi, \Psi\right\rangle} \\
\dot{\gamma} & =\frac{\left\langle j \mathbf{P}^{0}\left(\partial_{\omega} \Phi_{\omega}-\partial_{\omega} \mathbf{P}^{0} \chi\right), \mathbf{P}^{0} \mathbf{Q}\right\rangle}{\left\langle\partial_{\omega} \Phi_{\omega}-\partial_{\omega} \mathbf{P}^{0} \chi, \Psi\right\rangle}, .
\end{aligned}
$$

where $\mathbf{P}^{0}=\mathbf{P}^{0}(\omega(t))$ is the projection operator defined in (6.5) and $\partial_{\omega} \mathbf{P}^{0}=\partial_{\omega} \mathbf{P}^{0}(\omega)$ evaluated at $\omega=\omega(t)$.

(ii) Conversely given $\psi$ a solution of (4.4) as in theorem 2.2 and continuously differentiable functions $\omega(t), \theta(t)$ which satisfy (9.7)-(9.8) then $\chi$ defined by (9.2) satisfies (9.6) and the condition (9.3) holds at all times if it holds initially. 
Proof This can be proved as in [5, Prop.2.2].

It remains to show, for appropriate initial data close to a soliton, that there exist solutions to (9.7)-(9.8), at least locally. To achieve this observe that if the spectral condition (3.3) holds then by Lemma 3.4 the denominator appearing on the right hand side of (9.7) and (9.8) does not vanish for small $\|\chi\|_{L_{\beta}^{1}}$. This is because

$$
\left\langle\partial_{\omega} \psi_{s}, \psi_{s}\right\rangle=\frac{1}{2} \partial_{\omega} \int\left|\psi_{\omega}\right|^{2} d x \neq 0
$$

as discussed in section 3. This has the consequence that the orthogonality conditions really can be satisfied for small $\chi$ because they are equivalent to a locally well posed set of ordinary differential equations for $t \rightarrow(\theta(t), \omega(t))$. This implies the following corollary:

Corollary 9.2 (i) Under conditions of previous lemma assume that (9.9) holds. If $\|\chi\|_{L_{\beta}^{p}}$ is sufficiently small for some $p, \beta$ the right hand sides of (9.7) and (9.8) are smooth in $\theta, \omega$ and there exists continuous $\mathcal{R}=\mathcal{R}(\omega, \chi)$ such that

$$
|\dot{\gamma}(t)| \leq \mathcal{R}|\chi(0, t)|^{2}, \quad|\dot{\omega}(t)| \leq \mathcal{R}|\chi(0, t)|^{2} .
$$

(ii) Assume given $\psi$, a solution of (4.4) as in Theorem 2.2. If $\omega_{0}$ satisfies (9.9) and $\chi(x, 0)=$ $e^{-j \theta_{0}} \psi(x, 0)-\Phi_{\omega_{0}}(x)$ is small in some $L_{\beta}^{p}$ norm and satisfies (9.3) there is a time interval on which there exist $C^{1}$ functions $t \mapsto(\omega(t), \gamma(t))$ which satisfy (9.7)-(9.8).

\section{Time decay for the transversal dynamics}

In this section we state our Theorem 10.2 on the time decay of the transversal component $\chi(t)$ in the nonlinear setting, leaving the proof to the next section. Theorem 10.2 will be used to prove the main theorem in Section 12. First we represent the initial data $\psi_{0}$ in a convenient form for application of the modulation equations: the next Lemma will allow us to assume that (9.3) holds initially without loss of generality.

Lemma 10.1 Under conditions of Theorem 3.7 there exists a solitary wave $\psi_{\tilde{\omega}_{0}}=\tilde{C}_{0} e^{-\sqrt{\tilde{\omega}_{0}}|x|}$ satisfying the spectral condition (3.3) such that in vector form

$$
\psi_{0}(x)=e^{j \tilde{\theta}_{0}}\left(\Phi_{\tilde{\omega}_{0}}(x)+\chi_{0}(x)\right), \quad \Phi_{\tilde{\omega}_{0}}=\left(\psi_{\tilde{\omega}_{0}}, 0\right),
$$

and for $\chi_{0}(x)$ we have

$$
\mathbf{P}^{0}\left(\tilde{\omega}_{0}\right)\left(\chi_{0}\right)=0
$$

and

$$
\left\|\chi_{0}\right\|_{L_{\beta}^{1} \cap H^{1}}=\tilde{d}=O(d) \quad \text { as } d \rightarrow 0 .
$$

Proof By (9.4), the condition (10.1) is equivalent to the pair of equations

$$
\Omega\left(e^{-j \tilde{\theta}_{0}} \psi_{0}-\Phi_{\tilde{\omega}_{0}}, T_{0}\left(\tilde{\omega}_{0}\right)\right)=0, \quad \Omega\left(e^{-j \tilde{\theta}_{0}} \psi_{0}-\Phi_{\tilde{\omega}_{0}}, T_{1}\left(\tilde{\omega}_{0}\right)\right)=0,
$$

where $T_{0}(\omega)=j \Phi_{\omega}, T_{1}(\omega)=\partial_{\omega} \Phi_{\omega}$. For $\psi_{0}$ sufficiently close (in $L_{\beta}^{1}$ ) to $e^{j \theta_{0}} \Phi_{\omega_{0}}$ the existence of $\tilde{\theta}_{0}, \tilde{\omega}_{0}$ follows by a standard application of the implicit function theorem if we show that the Jacobian matrix

$$
\left(\begin{array}{cc}
\partial_{\omega} \Omega\left(e^{-j \theta} \psi_{0}-\Phi_{\omega}, j \Phi_{\omega}\right) & \partial_{\omega} \Omega\left(e^{-j \theta} \psi_{0}-\Phi_{\omega}, \partial_{\omega} \Phi_{\omega}\right) \\
\partial_{\theta} \Omega\left(e^{-j \theta} \psi_{0}-\Phi_{\omega}, j \Phi_{\omega}\right) & \partial_{\theta} \Omega\left(e^{-j \theta} \psi_{0}-\Phi_{\omega}, \partial_{\omega} \Phi_{\omega}\right)
\end{array}\right)
$$


with $\psi_{0}=e^{j \theta_{0}} \Phi_{\omega_{0}}$, is non-degenerate at $\omega=\omega_{0}$ and $\theta=\theta_{0}$. But this is equivalent to the non-degeneracy of the matrix

$$
\left(\begin{array}{cc}
\Omega\left(\partial_{\omega} \Phi_{\omega_{0}}, j \Phi_{\omega_{0}}\right) & 0 \\
0 & \Omega\left(j \Phi_{\omega_{0}}, \partial_{\omega} \Phi_{\omega_{0}}\right)
\end{array}\right)
$$

which holds by (9.9).

In Section 12 we will show that our main Theorem 3.7 can be derived from the following time decay of the transversal component $\chi(t)$ :

Theorem 10.2 Let all the assumptions of Theorem 3.7 hold. For d sufficiently small there exist $C^{1}$ functions $t \mapsto(\omega(t), \gamma(t))$ defined for $t \geq 0$ such that the solution $\psi(x, t)$ of (4.4) can be written as in (9.2-9.3) with (9.7-9.8) satisfied, and there exists a number $\bar{M}>0$, depending only on the initial data, such that

$$
M(T)=\sup _{0 \leq t \leq T}\left[(1+t)^{3 / 2}\|\chi(t)\|_{L_{-\beta}^{\infty}}+(1+t)^{3}(|\dot{\gamma}|+|\dot{\omega}|) \mid\right] \leq \bar{M},
$$

uniformly in $T>0$, and $\bar{M}=O(d)$ as $d \rightarrow 0$.

Remarks 10.3 (0) This theorem will be deduced from Proposition 11.1 in the next section.

(i) Theorem 2.2 implies that the norms in the definition of $M$ are continuous functions of time (and so $M$ is also).

(ii) The result holds also for negative time with appropriate changes since $\psi(x, t)$ solves (1.1) if and only if $\bar{\psi}(x,-t)$ does.

(iii) The result implies in particular that $t^{3}|\dot{\theta}-\omega|+t^{3}|\dot{\omega}| \leq C$, hence $\omega(t)$ and $\theta(t)-t \omega_{+}$ should converge as $t \rightarrow \infty$ while $\psi(x, t)-e^{j \theta(t)} \Phi_{\omega(t)}(x)$ have limit zero in $L_{-\beta}^{\infty}(\mathbb{R})$.

(iv) The notation $\chi(t)$ indicates the function $x \mapsto \chi(x, t)$ as usual.

\section{Proof of transversal decay}

\subsection{Inductive argument (proof of Theorem 10.2)}

Let us write the initial data in the form

$$
\psi_{0}(x)=e^{j \theta_{0}}\left(\Phi_{\omega_{0}}(x)+\chi_{0}(x)\right) .
$$

with $d=\left\|\chi_{0}\right\|_{L_{\beta}^{1} \cap H^{1}}$ sufficiently small. By Lemma 10.1 we can assume that $\mathbf{P}^{0}\left(\omega_{0}\right)\left(\chi_{0}\right)=$ 0 without loss of generality. Then the local existence asserted in Corollary 9.2 implies the existence of an interval $\left[0, t_{1}\right]$ on which are defined $C^{1}$ functions $t \mapsto(\omega(t), \gamma(t))$ satisfying (9.7)-(9.8) and such that $M\left(t_{1}\right)=\rho$ for some $t_{1}>0$ and $\rho>0$. By continuity we can make $\rho$ as small as we like by making $d$ and $t_{1}$ small. The following Proposition is proved in section 11.4 below.

Proposition 11.1 Under conditions of Theorem 10.2 let $M\left(t_{1}\right) \leq \rho$ for some $t_{1}>0$ and $\rho>0$. Then there exist numbers $d_{1}$ and $\rho_{1}$, independent of $t_{1}$, such that

$$
M\left(t_{1}\right) \leq \rho / 2
$$

if $d=\left\|\chi_{0}\right\|_{L_{\beta}^{1} \cap H^{1}}<d_{1}$ and $\rho<\rho_{1}$. 
Assuming the truth of Proposition 11.1 for now Theorem 10.2 will follow from the next argument:

Consider the set $\mathcal{T}$ of $t_{1} \geq 0$ such that $(\omega(t), \gamma(t))$ are defined on $\left[0, t_{1}\right]$ and $M\left(t_{1}\right) \leq \rho$. This set is relatively closed by continuity. On the other hand, (11.2) and Corollary 9.2 with sufficiently small $\rho$ and $d$ imply that this set is also relatively open, and hence $\sup \mathcal{T}=+\infty$, completing the proof of Theorem 10.2.

\subsection{Frozen linearized equation}

A crucial part of the proof of Proposition 11.1 is the estimation of the first term in $M$, for which purpose it is necessary to make use of the dispersive properties obtained in sections 6 and 7. Rather than study directly (9.6), whose linear part is non-autonomous, it is convenient (following $[2,3]$ ) to introduce a second ansatz, a small modification of (9.2), which leads to an autonomous linearized equation. This new ansatz for the solution is

$$
\psi(x, t)=e^{j \theta}\left(\Phi_{\omega}(x)+e^{-j(\theta-\tilde{\theta})} \eta\right), \quad \text { where } \tilde{\theta}(t)=\omega_{1} t+\theta_{0}, \theta_{0}=\theta_{0} \text { and } \omega_{1}=\omega\left(t_{1}\right)
$$

so that, $\eta=e^{j(\theta-\tilde{\theta})} \chi$ and $\chi=e^{-j(\theta-\tilde{\theta})} \eta$. Since

$$
\dot{\chi}=e^{-j(\theta-\tilde{\theta})}\left(\dot{\eta}-j\left(\omega+\dot{\gamma}-\omega_{1}\right) \eta\right)
$$

equation (9.6) implies

$$
\dot{\eta}=j^{-1}\left(\omega_{1}-\omega\right) \eta+e^{j(\theta-\tilde{\theta})} \mathbf{C}\left(e^{-j(\theta-\tilde{\theta})} \eta\right)+e^{j(\theta-\tilde{\theta})}\left(j^{-1} \dot{\gamma} \Phi_{\omega}-\dot{\omega} \partial_{\omega} \Phi_{\omega}+\mathbf{Q}\left[e^{-j(\theta-\tilde{\theta})} \eta\right]\right) .
$$

The matrices $\mathbf{C}$ and $e^{j \phi}$, where $\phi=\theta-\tilde{\theta}$, do not commute hence we need the following lemma:

Lemma 11.2 The following commutation relation holds,

$$
\mathbf{C} e^{j \phi}-e^{j \phi} \mathbf{C}=\delta(x) b \sin \phi \sigma, \text { where } \sigma=\left(\begin{array}{cc}
1 & 0 \\
0 & -1
\end{array}\right), b=2 a^{\prime} C^{2} .
$$

Proof

$$
\begin{gathered}
\mathbf{C} e^{j \phi}-e^{j \phi} \mathbf{C}=\left(\begin{array}{cc}
0 & \mathbf{D}_{2} \\
-\mathbf{D}_{1} & 0
\end{array}\right)\left(\begin{array}{cc}
\cos \phi & -\sin \phi \\
\sin \phi & \cos \phi
\end{array}\right)-\left(\begin{array}{cc}
\cos \phi & -\sin \phi \\
\sin \phi & \cos \phi
\end{array}\right)\left(\begin{array}{cc}
0 & \mathbf{D}_{2} \\
-\mathbf{D}_{1} & 0
\end{array}\right) \\
=\left(\begin{array}{cc}
\left(\mathbf{D}_{2}-\mathbf{D}_{1}\right) \sin \phi & 0 \\
0 & \left(\mathbf{D}_{1}-\mathbf{D}_{2}\right) \sin \phi
\end{array}\right)=\left(\begin{array}{cc}
\delta(x) b \sin \phi & 0 \\
0 & -\delta(x) b \sin \phi
\end{array}\right) .
\end{gathered}
$$

Using Lemma 11.2 we rewrite equation (11.4) as

$$
\dot{\eta}=j^{-1}\left(\omega_{1}-\omega\right) \eta+\mathbf{C} \eta+e^{j(\theta-\tilde{\theta})}\left(-\delta(x) b \sin (\theta-\tilde{\theta}) \sigma \eta+j^{-1} \dot{\gamma} \Phi_{\omega}-\dot{\omega} \partial_{\omega} \Phi_{\omega}+\mathbf{Q}\left[e^{-j(\theta-\tilde{\theta})} \eta\right]\right) .
$$

To obtain a perturbed autonomous equation we rewrite the first two terms on the RHS by freezing the coefficients at $t=t_{1}$. Note that

$$
j^{-1}\left(\omega_{1}-\omega\right)+\mathbf{C}=\mathbf{C}_{\mathbf{1}}-j^{-1} \delta(x)\left(V-V_{1}\right),
$$

where $V=a+b P_{1}, V_{1}=V\left(t_{1}\right)$, and $\mathbf{C}_{1}=\mathbf{C}\left(t_{1}\right)$. The equation for $\eta$ now reads

$$
\dot{\eta}=\mathbf{C}_{\mathbf{1}} \eta-j^{-1} \delta(x)\left(V-V_{1}\right) \eta+e^{j(\theta-\tilde{\theta})}\left(-\delta(x) b \sin (\theta-\tilde{\theta}) \sigma \eta+j^{-1} \dot{\gamma} \Phi-\dot{\omega} \partial_{\omega} \Phi_{\omega}+\mathbf{Q}\left[e^{-j(\theta-\tilde{\theta})} \eta\right]\right)
$$


The first term is now independent of $t$; the idea is that if there is sufficiently rapid convergence of $\omega(t)$ as $t \rightarrow \infty$ the other remaining terms are small uniformly with respect to $t_{1}$. Finally the equation (11.6) can be written in the following frozen form

$$
\dot{\eta}=\mathbf{C}_{1} \eta+\mathbf{f}_{1}
$$

where

$$
\mathbf{f}_{\mathbf{1}}=-j^{-1} \delta(x)\left(V-V_{1}\right) \eta+e^{j(\theta-\tilde{\theta})}\left(-\delta(x) b \sin (\theta-\tilde{\theta}) \sigma \eta+j^{-1} \dot{\gamma} \Phi-\dot{\omega} \partial_{\omega} \Phi_{\omega}+\mathbf{Q}\left[e^{-j(\theta-\tilde{\theta})} \eta\right]\right)
$$

Remark 11.3 The advantage of (11.7) over (9.6) is that it can be treated as a perturbed autonomous linear equation, so that the estimates from section 6 can be used directly. The additional terms in $\mathbf{f}_{\mathbf{1}}$ can be estimated as small uniformly in $t_{1}$ : see lemma 11.4 below. This is the reason for introduction of the second ansatz (11.3).

Lemma 11.4 Under conditions of Proposition 11.1 there exists $c>0$, independent of $t_{1}$, such that for $0 \leq t \leq t_{1}$

$$
\left|a(t)-a_{1}\right|+\left|b(t)-b_{1}\right|+|\theta(t)-\tilde{\theta}(t)| \leq c \rho .
$$

Proof By (10.4)

$$
\sup _{0 \leq t \leq t_{1}}\left(1+t^{3}\right)(|\dot{\gamma}(t)|+|\dot{\omega}(t)|) \leq M\left(t_{1}\right)=\rho
$$

Therefore

$$
\left|a(t)-a\left(t_{1}\right)\right|=\left|\int_{t}^{t_{1}} \dot{a}(\tau) d \tau\right| \leq c\left(\sup _{0 \leq \tau \leq t_{1}}\left(1+\tau^{2}\right)|\dot{\omega}(\tau)|\right) \int_{t}^{t_{1}} \frac{d \tau}{1+\tau^{2}} \leq c \rho,
$$

since $|\dot{a}(\tau)| \leq c|\dot{\omega}(\tau)|$. The difference $\left|b(t)-b\left(t_{1}\right)\right|$ can be estimated similarly. Next

$$
\begin{aligned}
\theta(t)-\tilde{\theta}(t) & =\int_{0}^{t} \omega(\tau) d \tau+\gamma(t)-\omega\left(t_{1}\right) t-\gamma(0)=\int_{0}^{t}\left(\omega(\tau)-\omega\left(t_{1}\right)\right) d \tau+\int_{0}^{t} \dot{\gamma}(\tau) d(\mathcal{A} 1 \\
& =-\int_{0}^{t} \int_{\tau}^{t_{1}} \dot{\omega}(s) d s d \tau+\int_{0}^{t} \dot{\gamma}(\tau) d \tau
\end{aligned}
$$

By (11.9) the first summand in RHS of (11.10) can be estimated as

$$
\begin{gathered}
\int_{0}^{t} \int_{t_{1}}^{\tau}|\dot{\omega}(s)| d s d \tau \leq \int_{0}^{t} \int_{\tau}^{t_{1}}(1+s)^{2+\varepsilon}|\dot{\omega}(s)| \frac{1}{(1+s)^{2+\varepsilon}} d s d \tau \\
\quad \leq c \sup _{0 \leq s \leq t_{1}}(1+s)^{2+\varepsilon}|\dot{\omega}(s)| \int_{0}^{t} \int_{\tau}^{t_{1}} \frac{1}{(1+s)^{2+\varepsilon}} d s d \tau \leq c \rho
\end{gathered}
$$

since the last integral is bounded for $t \in\left[0, t_{1}\right]$. Finally, for the second summand on the RHS of (11.10) inequality (11.9) implies

$$
\left|\int_{0}^{t} \dot{\gamma}(\tau) d \tau\right| \leq c \sup _{0 \leq \tau \leq t_{1}}\left(1+\tau^{2}\right)|\dot{\gamma}(\tau)| \int_{t}^{t_{1}} \frac{d \tau}{1+\tau^{2}} \leq c \rho
$$




\subsection{Projection onto discrete and continuous spectral spaces}

¿From sections 6 and 7 we have information concerning $U(t)=e^{\mathbf{C}_{\mathbf{1}} t}$, in particular decay on the subspace orthogonal to the (two dimensional) generalized null space. It is therefore necessary to introduce a further decomposition to take advantage of this. Recall, by comparing (9.2) and (11.3) that

$$
\eta=e^{j(\theta-\tilde{\theta})} \chi \quad \text { and } \quad \mathbf{P}_{t}^{0} \chi(t)=0
$$

Introduce the symplectic projections $\mathbf{P}_{1}^{0}=\mathbf{P}_{t_{1}}^{0}$ and $\mathbf{P}_{1}^{c}=\mathbf{P}_{t_{1}}^{c}$ onto the discrete and continuous spectral subspaces defined by the operator $\mathbf{C}_{\mathbf{1}}$ and write, at each time $t \in\left[0, t_{1}\right]$ :

$$
\eta(t)=g(t)+h(t)
$$

with $g(t)=\mathbf{P}_{1}^{0} \eta(t)$ and $h(t)=\mathbf{P}_{1}^{c} \eta(t)$. The following lemma shows that it is only necessary to estimate $h(t)$.

Lemma 11.5 Under conditions of Proposition 11.1, assume

$$
\sup _{0 \leq t \leq t_{1}}\left(\left|\omega(t)-\omega_{1}\right|+\left|\theta(t)-\theta_{1}(t)\right|\right)=\Delta
$$

is sufficiently small. Then for $0 \leq t \leq t_{1}$ there exists a linear operator $\Xi(t)$, bounded on $L_{-\beta}^{\infty} \cap H^{1}$, and $c\left(\Delta, \omega_{1}\right)$ such that $\eta(t)=\Xi(t) h(t)$, and

$$
c\left(\Delta, \omega_{1}\right)^{-1}\left|\|h\|_{L_{-\beta}^{\infty} \cap H^{1}} \leq\|\eta\|_{L_{-\beta}^{\infty} \cap H^{1}} \leq c\left(\Delta, \omega_{1}\right)\right|\|h\|_{L_{-\beta}^{\infty} \cap H^{1}} .
$$

Proof Explicitly we write

$$
\eta(t)=h(t)+g(t), \quad g(t)=b_{0}(t) T_{0}\left(\omega_{1}\right)+b_{1}(t) T_{1}\left(\omega_{1}\right)
$$

where $b_{0}, b_{1}$ are chosen at each time $t$ to ensure that $\Omega\left(h(t), T_{0}\left(\omega_{1}\right)\right)=\Omega\left(h(t), T_{1}\left(\omega_{1}\right)\right)=0$. Using the fact that (since $\left.\mathbf{P}_{t}^{0} \chi(t)=0\right)$

$$
\Omega\left(e^{-j(\theta-\tilde{\theta})} \eta, T_{0}(\omega(t))=0=\Omega\left(e^{-j(\theta-\tilde{\theta})} \eta, T_{1}(\omega(t))\right.\right.
$$

this means that $b_{0}, b_{1}$ are determined by

$$
\begin{aligned}
-\boldsymbol{\mu}_{\boldsymbol{\omega}_{\mathbf{1}}} b_{0}(t) & =\Omega\left(\eta(t), T_{1}\left(\omega_{1}\right)\right)=\Omega\left(\eta(t), T_{1}\left(\omega_{1}\right)-e^{j(\theta-\tilde{\theta})} T_{1}(\omega(t))\right) \\
\boldsymbol{\mu}_{\boldsymbol{\omega}_{\mathbf{1}}} b_{1}(t) & =\Omega\left(\eta(t), T_{0}\left(\omega_{1}\right)\right)=\Omega\left(\eta(t), T_{0}\left(\omega_{1}\right)-e^{j(\theta-\tilde{\theta})} T_{0}(\omega(t))\right) .
\end{aligned}
$$

From these it follows that there exists $c>0$ such that $\|g(t)\|_{L_{-\beta}^{\infty} \cap H^{1}} \leq c \Delta\|\eta(t)\|_{L_{-\beta}^{\infty} \cap H^{1}}$ and hence (11.13) follows as claimed.

\subsection{Proof of Proposition 11.1}

To prove Proposition 11.1 we explain how to estimate both terms in $M$, (10.4), to be $\leq \rho / 4$, uniformly in $t_{1}$.

Estimation of the second term in $M$. As in Corollary 9.2 we have

$$
|\dot{\gamma}(t)|+|\dot{\omega}(t)| \leq c_{0}|\chi(0, t)|^{2} \leq c_{0} \frac{M(t)^{2}}{(1+|t|)^{3}}, \quad t \leq t_{1},
$$


since $|\chi(0, t)| \leq\|\chi(t)\|_{L_{-\beta}^{\infty}}$. Finally let $\rho_{1}<1 /\left(4 c_{0}\right)$ to complete the estimate for the second term in $M$ as $\leq \rho / 4$.

Estimation of the first term in $M$. By Lemma 11.5 it is enough to estimate $h$. Let us apply the projection $\mathbf{P}_{1}^{c}$ to both sides of (11.7). Then the equation for $h$ reads

$$
\dot{h}=\mathbf{C}_{1} h+\mathbf{P}_{1}^{\mathbf{c}} \mathbf{f}_{\mathbf{1}}
$$

Now to estimate $h$ we use the Duhamel representation:

$$
h(t)=U(t) h(0)+\int_{0}^{t} U(t-s) \mathbf{P}_{1}^{c} \mathbf{f}_{\mathbf{1}}(s) d s, \quad t \leq t_{1} .
$$

with $U(t)=e^{\mathbf{C}_{1} t}$ the one parameter group just introduced. Recall that $\mathbf{P}_{1}^{0} h(t)=0$ for $t \in\left[0, t_{1}\right]$. Therefore

$$
\|U(t) h(0)\|_{L_{-\beta}^{\infty}} \leq c(1+t)^{-3 / 2}\|h(0)\|_{L_{\beta}^{1} \cap H^{1}} \leq c(1+t)^{-3 / 2}\|\eta(0)\|_{L_{\beta}^{1} \cap H^{1}} .
$$

by Theorem 7.1 and inequalities (8.1) and (11.13). Let us estimate the integrand on the righthand side of (11.18).

Lemma 11.6 The integrand in (11.18) satisfies the following bound:

$$
\left\|U(t-s) \mathbf{P}_{1}^{c} \mathbf{f}_{\mathbf{1}}(\mathbf{s})\right\|_{L_{-\beta}^{\infty}} \leq c \frac{1}{(t-s)^{1 / 2}(1+t-s)}\left(\|\eta(s)\|_{L_{-\beta}^{\infty}}^{2}+\rho\|\eta(s)\|_{L_{-\beta}^{\infty}}\right), \quad 0<s<t .
$$

Proof We consider two different cases : $t-s>\nu$, and $0<t-s<\nu$, where $\nu=\nu(a, b)$ is defined in Lemma 8.1.

i) $t-s>\nu$ : We use the representation (11.8) for $\mathbf{f}_{\mathbf{1}}$ and apply Theorem 7.1, Corollary 9.2 and Lemma 11.4 to obtain that

$$
\begin{aligned}
\left\|U(t-s) \mathbf{P}_{1}^{c} \mathbf{f}_{\mathbf{1}}\right\|_{L_{-\beta}^{\infty}} & \leq c(\nu)(1+t-s)^{-3 / 2}\left\|\mathbf{P}_{1}^{c}\left(\mathbf{f}_{\mathbf{1}}(t)\right)\right\|_{\mathcal{M}} \\
& \leq c(\nu)(1+t-s)^{-3 / 2}\left(|\eta(0, t)|^{2}+\rho|\eta(0, t)|\right) \\
& \leq c(\nu)(1+t-s)^{-3 / 2}\left(\|\eta(t)\|_{L_{-\beta}^{\infty}}^{2}+\rho\|\eta(t)\|_{L_{-\beta}^{\infty}}\right), \quad t \leq t_{1} .
\end{aligned}
$$

ii) $0<t-s<\nu$ : We denote $\mathbf{Q}=\delta(x) \tilde{\mathbf{Q}}$, and represent $\mathbf{f}_{\mathbf{1}}(x, s)$ as

$$
\mathbf{f}_{\mathbf{1}}(x, s)=p(s) \delta(x)+q(s) \Phi_{\omega}+r(s) \partial_{\omega} \Phi_{\omega} .
$$

where

$$
p(s)=-j^{-1}\left(V-V_{1}\right) \eta(0, s)+e^{j(\theta-\tilde{\theta})}\left(b \sin (\theta-\tilde{\theta}) \sigma \eta(0, s)+\tilde{\mathbf{Q}}\left[e^{-j(\theta-\tilde{\theta})} \eta(0, s)\right]\right),
$$

is an $\mathbb{R}^{2}$ valued function of time, and

$$
q(s)=-e^{-j(\theta-\tilde{\theta})} j \dot{\gamma}, \quad r(s)=-e^{-j(\theta-\tilde{\theta})} \dot{\omega}
$$

are $(2 \times 2)$ matrix valued functions of time. Writing $\|\cdot\|$ for both the standard Euclidean and operator norms on these, we have, by Lemma 11.4,

$$
\|p(s)\| \leq c\left(|\eta(0, s)|^{2}+\rho|\eta(0, s)|\right) \leq c\left(\|\eta(s)\|_{L_{-\beta}^{\infty}}^{2}+\rho\|\eta(s)\|_{L_{-\beta}^{\infty}}\right)
$$


and by Corollary 9.2

$$
\|q(s)\|,\|r(s)\| \leq c|\eta(0, s)|^{2} \leq c\|\eta(s)\|_{L_{-\beta}^{\infty}}^{2} .
$$

Applying projector $\mathbf{P}_{1}^{c}$ to $\mathbf{f}_{\mathbf{1}}$ we obtain

$$
\mathbf{P}_{1}^{c} \mathbf{f}_{\mathbf{1}}(x, s)=p(s) \delta(x)+q(s) \Phi_{\omega}+r(s) \partial_{\omega} \Phi_{\omega}-\mathbf{P}_{1}^{0} \mathbf{f}_{\mathbf{1}}(x, s) .
$$

By Lemma 8.1 for sufficiently small $\nu$ we obtain

$$
\begin{aligned}
& \|U(t-s) p(s) \delta(x)\|_{L_{-\beta}^{\infty}} \leq\|U(t-s) p(s) \delta(x)\|_{L^{\infty}} \leq c(\nu)\|p(s)\|(t-s)^{-1 / 2} \\
\leq & c(\nu)(t-s)^{-1 / 2}\left(\|\eta(s)\|_{L_{-\beta}^{\infty}}^{2}+\rho\|\eta(s)\|_{L_{-\beta}^{\infty}}\right), \quad 0<t-s<\nu .
\end{aligned}
$$

By inequality (8.1) we have

$$
\begin{aligned}
& \left\|\quad U(t-s)\left(q(s) \Phi_{\omega}+r(s) \partial_{\omega} \Phi_{\omega}\right)\right\|_{L_{-\beta}^{\infty}} \leq c\left\|U(t-s)\left(q(s) \Phi_{\omega}+r(s) \partial_{\omega} \Phi_{\omega}\right)\right\|_{H^{1}} \\
& \leq c\left(\|q(s)\|\left\|\Phi_{\omega}\right\|_{H^{1}}+\left(\|r(s)\|\left\|\partial_{\omega} \Phi_{\omega}\right\|_{H^{1}}\right) \leq c\|\eta(s)\|_{L_{-\beta}^{\infty}}^{2}, \quad 0 \leq t-s<\nu\right.
\end{aligned}
$$

The definition (6.5) of the projector $\mathbf{P}_{1}^{0}$ implies immediately that

$$
\left\|\mathbf{P}_{1}^{0} \mathbf{f}_{\mathbf{1}}\right\|_{H^{1}} \leq c(\|p(s)\|+\|q(s)\|+\|r(s)\|)
$$

Then, similarly to (11.25), we obtain

$$
\left\|U(t-s) \mathbf{P}_{1}^{0} \mathbf{f}_{\mathbf{1}}\right\|_{L_{-\beta}^{\infty}} \leq c\left(\|\eta(s)\|_{L_{-\beta}^{\infty}}^{2}+\rho\|\eta(s)\|_{L_{-\beta}^{\infty}}\right), \quad 0 \leq t-s<\nu
$$

Finally, (11.23)-(11.26) imply

$$
\left\|U(t-s) \mathbf{P}_{1}^{c} \mathbf{f}_{\mathbf{1}}\right\|_{L_{-\beta}^{\infty}} \leq c(t-s)^{-1 / 2}\left(\|\eta(s)\|_{L_{-\beta}^{\infty}}^{2}+\rho\|\eta(s)\|_{L_{-\beta}^{\infty}}\right), \quad 0<t-s<\nu .
$$

From (11.21) and (11.27) inequality (11.20) follows.

Now (11.13), (11.18), (11.19) and (11.20) imply

$$
\|\eta(t)\|_{L_{-\beta}^{\infty}} \leq c(1+t)^{-3 / 2}\|\eta(0)\|_{L_{\beta}^{1} \cap H^{1}}+c_{1} \int_{0}^{t} \frac{d s}{(t-s)^{1 / 2}(1+t-s)}\left(\|\eta(s)\|_{L_{-\beta}^{\infty}}^{2}+\rho\|\eta(s)\|_{L_{-\beta}^{\infty}}\right)
$$

Multiply by $(1+t)^{3 / 2}$ to deduce

$$
\begin{aligned}
(1+t)^{3 / 2}\|\eta(t)\|_{L_{-\beta}^{\infty}} \leq c d & +c_{1} \int_{0}^{t} \frac{(1+t)^{3 / 2}(1+s)^{-3}}{(t-s)^{1 / 2}(1+t-s)}(1+s)^{3}\|\eta(s)\|_{L_{-\beta}^{\infty}}^{2} d s \\
& +c_{1} \rho \int_{0}^{t} \frac{(1+t)^{3 / 2}(1+s)^{-3 / 2}}{(t-s)^{1 / 2}(1+t-s)}(1+s)^{3 / 2}\|\eta(s)\|_{L_{-\beta}^{\infty}} d s
\end{aligned}
$$

since $\|\eta(0)\|_{L_{\beta}^{1} \cap H^{1}} \leq d$. Introduce the majorant

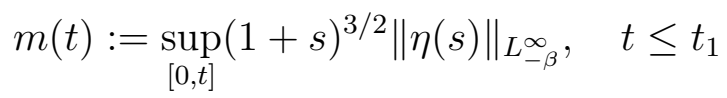


and hence

$$
m(t) \leq c d+c_{1} m^{2}(t) \int_{0}^{t} \frac{(1+t)^{3 / 2}(1+s)^{-3}}{(t-s)^{1 / 2}(1+t-s)} d s+\rho c_{1} m(t) \int_{0}^{t} \frac{(1+t)^{3 / 2}(1+s)^{-3 / 2}}{(t-s)^{1 / 2}(1+t-s)} d s .
$$

It easy to see (by splitting up the integrals into $s<t / 2$ and $s \geq t / 2$ ) that both these integrals are bounded independent of $t$. Thus (11.29) implies that there exist $c, c_{2}, c_{3}$, independent of $t_{1}$, such that

$$
m(t) \leq c d+\rho c_{2} m(t)+c_{3} m^{2}(t), \quad t \leq t_{1} .
$$

Recall that $m\left(t_{1}\right) \leq \rho \leq \rho_{1}$ by assumption. Therefore this inequality implies that $m(t)$ is bounded for $t \leq t_{1}$, and moreover,

$$
m(t) \leq c_{4} d, \quad t \leq t_{1}
$$

if $d$ and $\rho$ are sufficiently small. The constant $c_{4}$ does not depend on $t_{1}$. We choose $d$ in (3.4) small enough that $d<\rho /\left(4 c_{4}\right)$. Therefore,

$$
\sup _{\left[0, t_{1}\right]}(1+t)^{3 / 2}\|\eta(t)\|_{L_{-\beta}^{\infty}}<\rho / 4
$$

if $d$ and $\rho$ are sufficiently small. This bounds the first term as $<\rho / 4$ by (11.11) and hence $M\left(t_{1}\right)<\rho / 2$, completing the proof of Proposition 11.1.

\section{Soliton asymptotics}

Here we prove our main Theorem 3.7 using the bounds (10.4). For the solution $\psi(x, t)$ to (1.1) let us define the accompanying soliton as $s(x, t)=\psi_{\omega(t)}(x) e^{i \theta(t)}$, where $\dot{\theta}(t)=\omega(t)+\dot{\gamma}(t)$. Then for the difference $z(x, t)=\psi(x, t)-s(x, t)$ we obtain easily from equations (1.1) and (2.7)

$$
i \dot{z}(x, t)=-z^{\prime \prime}(x, t)+\dot{\gamma} s(x, t)-i \dot{\omega} \partial_{\omega} s(x, t)-\delta(x)(F(\psi(x, t))-F(s(x, t))) .
$$

Then

$$
z(t)=W(t) z(0)+\int_{0}^{t} W(t-\tau)\left[\dot{\gamma} s(\tau)-i \dot{\omega} \partial_{\omega} s(\tau)-\delta(x)(F(\psi(0, \tau))-F(s(0, \tau)))\right] d \tau,
$$

where $z(t)=z(\cdot, t), s(t)=s(\cdot, t)$, and $W(t)$ is the dynamical group of the free Schrödinger equation. Since $\gamma(t)-\gamma_{+}, \omega(t)-\omega_{+}=\mathcal{O}\left(t^{-2}\right)$, and therefore $\theta(t)-\omega_{+} t-\gamma_{+}=\mathcal{O}\left(t^{-1}\right)$ for $t \rightarrow \infty$, to establish the asymptotic behaviour (3.5) it suffices to prove that

$$
z(t)=W(t) \Phi_{+}+r_{+}(t)
$$

with some $\Phi_{+} \in C_{b}(\mathbb{R}) \cap L^{2}(\mathbb{R})$ and $\left\|r_{+}(t)\right\|_{C_{b}(\mathbb{R}) \cap L^{2}(\mathbb{R})}=\mathcal{O}\left(t^{-1 / 2}\right)$. Denote $g(t)=\dot{\gamma} s(t)-$ $i \dot{\omega} \partial_{\omega} s(t), h(t)=F(\psi(0, t))-F(s(0, t))$ and rewrite equation (12.2) as

$$
z(t)=W(t) z(0)+W(t) \int_{0}^{t} W(-\tau) g(\tau) d \tau-W(t) \int_{0}^{t} W(-\tau) \delta(x) h(\tau) d \tau .
$$


Let us recall that $\dot{\omega}(t), \dot{\gamma}(t) \sim t^{-3}$ as $t \rightarrow \infty$. Hence, for the second summand in RHS of (12.4) we have

$$
W(t) \int_{0}^{t} W(-\tau) g(\tau) d \tau=W(t) \int_{0}^{\infty} W(-\tau) g(\tau) d \tau-W(t) \int_{t}^{\infty} W(-\tau) g(\tau) d \tau=W(t) \phi_{1}+r_{1}(t)
$$

where, from the unitarity in $H^{1}$ of the group $W(t)$ and the $t^{-3}$ decay of $\dot{\omega}$ and $\dot{\gamma}$, we infer that $\phi_{1}=\int_{0}^{\infty} W(-\tau) g(\tau) d \tau \in H^{1}$, and $\left\|r_{1}(t)\right\|_{H^{1}}=\mathcal{O}\left(t^{-2}\right), t \rightarrow \infty$.

Consider now the last summand in the RHS of (12.4). Note that $W(t) \delta(x)=\frac{e^{i x^{2} /(4 t)}}{\sqrt{4 \pi i t}}$, and $|h(t)| \leq c|\chi(0, t)| \leq c(1+t)^{-3 / 2}$ by (10.4). Therefore

$W(t) \int_{0}^{t} W(-\tau) \delta(x) h(\tau) d \tau=W(t) \int_{0}^{\infty} \frac{e^{-i x^{2} /(4 \tau)}}{\sqrt{-4 \pi i \tau}} h(\tau) d \tau-\int_{t}^{\infty} \frac{e^{i x^{2} /(4(t-\tau))}}{\sqrt{4 \pi i(t-\tau)}} h(\tau) d \tau=W(t) \phi_{2}+r_{2}(t)$.

Evidently, $\phi_{2}=\int_{0}^{\infty} \frac{e^{-i x^{2} /(4 \tau)}}{\sqrt{-4 \pi i \tau}} h(\tau) d \tau \in C_{b}$, and $\left\|r_{2}(t)\right\|_{C_{b}}=\mathcal{O}\left(t^{-1}\right), t \rightarrow \infty$.

Moreover, $\phi_{2} \in L^{2}$, and $\left\|r_{2}(t)\right\|_{L^{2}}=\mathcal{O}\left(t^{-1 / 2}\right), t \rightarrow \infty$. To see that this is indeed true change variable to $\tau=1 / u$ in the definition to get:

$$
\phi_{2}(x)=\frac{1}{\sqrt{-4 \pi i}} \int_{0}^{\infty} e^{-i u x^{2} / 4} \eta(u) d u, \quad \eta(u)=h(1 / u) / u^{3 / 2} .
$$

Now $h(t)$ is bounded and it follows from the decay of $h(t)$ that $\eta(u)$ is bounded as $u \rightarrow 0$. Therefore $\eta(u)$ is square integrable and so by the Parseval-Plancherel theorem $\phi_{2}$ is square integrable as a function of $y=x^{2}$, and hence also as a function of $x$ (since $d y=2 x d x$ and $\phi_{2}$ is a bounded continuous function). Next we have $r_{2}(t)=-W(t) R(t)$ with

$$
R(x, t)=\frac{1}{\sqrt{-4 \pi i}} \int_{0}^{1 / t} e^{-i u x^{2} / 4} \eta(u) d u=\frac{1}{\sqrt{-4 \pi i}} F_{u \rightarrow x^{2} / 4} \zeta_{t}(u) \eta(u),
$$

where $\zeta_{t}(u)$ is the characteristic function of the interval $(0,1 / t)$. The function $\eta(u)$ is bounded, hence $\left\|\zeta_{t} \eta\right\|_{L^{2}}=c t^{-1 / 2}$ and therefore $\left\|r_{2}(t)\right\|_{L^{2}}=\mathcal{O}\left(t^{-1 / 2}\right), t \rightarrow \infty$. To conclude, using (12.4), (12.5), and (12.6) we obtain (12.3) with $\phi_{+}=z(0)+\phi_{1}+\phi_{2}$ and $r_{+}(t)=r_{1}(t)+r_{2}(t)$. The $t \rightarrow-\infty$ case is handled in an identical way.

Remark 12.1 The expression (12.7) for $\phi_{2}$ as a Fourier transform implies immediately that $\left|\phi_{2}\right|$, and hence $\left|\Phi_{+}\right|$also, tend to 0 as $|x| \rightarrow \infty$ by the Riemann-Lebesgue lemma. This same expression could be used with Zygmund's lemma to obtain more detailed decay properties of $\phi_{2}$ and hence of $\Phi_{+}$. The decay rate would be determined essentially by the regularity of the function $h(t)$ in addition to the decay rate of the initial data.

\section{A The resolvent}

\section{A1 Calculation of the matrix kernel}

The derivation of the time decay of the solution to the linearized equation (4.2) in section 6 required an analysis of the smoothness and singularities of the resolvent $\mathbf{R}(\lambda)$ and its asymptotics 
for $\lambda \rightarrow \infty$. Here we will construct its matrix integral kernel explicitly

$$
\mathbf{R}(\lambda, x, y)=\left(\begin{array}{ll}
R_{11}(\lambda, x, y) & R_{12}(\lambda, x, y) \\
R_{21}(\lambda, x, y) & R_{22}(\lambda, x, y)
\end{array}\right)
$$

which is the solution to the equation

$$
(\mathbf{C}-\lambda) \mathbf{R}(\lambda, x, y)=\delta(x-y)\left(\begin{array}{ll}
1 & 0 \\
0 & 1
\end{array}\right) .
$$

Calculation of first column For the first column $R_{I}(\lambda, x, y):=\left(\begin{array}{c}R_{11}(\lambda, x, y) \\ R_{21}(\lambda, x, y)\end{array}\right)$ of the matrix $\mathbf{R}(\lambda, x, y)$ we obtain

$$
(\mathbf{C}-\lambda) R_{I}(\lambda, x, y)=\delta(x-y)\left(\begin{array}{l}
1 \\
0
\end{array}\right) .
$$

If $x \neq 0$ and $x \neq y$, (A. 3) takes the form (cf. (4.8), (4.9))

$$
\left(\begin{array}{ll}
-\lambda & \mathbf{D}_{2} \\
-\mathbf{D}_{1} & -\lambda
\end{array}\right) R_{I}(\lambda, x, y)=\left(\begin{array}{rr}
-\lambda & -\frac{d^{2}}{d x^{2}}+\omega \\
\frac{d^{2}}{d x^{2}}-\omega & -\lambda
\end{array}\right) R_{I}(\lambda, x, y)=0, \quad x \neq 0, \quad x \neq y .
$$

The general solution is a linear combination of exponential solutions of type $e^{i k x} v$. Substituting into (A. 4), we get

$$
\left(\begin{array}{rr}
-\lambda & k^{2}+\omega \\
-k^{2}-\omega & -\lambda
\end{array}\right) v=0
$$

For nonzero vectors $v$, the determinant of the matrix vanishes,

$$
\lambda^{2}+\left(k^{2}+\omega\right)^{2}=0 .
$$

Then $k_{ \pm}^{2}+\omega=\mp i \lambda$. Finally, we obtain four roots $\pm k_{ \pm}(\lambda)$ with

$$
k_{ \pm}(\lambda)=\sqrt{-\omega \mp i \lambda}
$$

where the square root is defined as an analytic continuation from a neighborhood of the zero point $\lambda=0$ taking the positive value of $\operatorname{Im} \sqrt{-\omega}$ at $\lambda=0$. We choose the cuts in the complex plane $\lambda$ from the branching points to infinity: the cut $\mathcal{C}_{+}:=[i \omega, i \infty)$ for $k_{+}(\lambda)$ and the cut $\mathcal{C}_{-}:=[-i \omega,-i \infty)$ for $k_{-}(\lambda)$. Then

$$
\operatorname{Im} k_{ \pm}(\lambda)>0, \quad \lambda \in \mathbb{C} \backslash \mathcal{C}_{ \pm}
$$

It remains to derive the vector $v=\left(v_{1}, v_{2}\right)$ which is solution to (A. 5):

$$
v_{2}=-\frac{k_{ \pm}^{2}+\omega}{\lambda} v_{1}=\frac{ \pm i \lambda}{\lambda} v_{1}= \pm i v_{1}
$$

Therefore, we have two corresponding vectors $v_{ \pm}=\left(\begin{array}{c}1 \\ \pm i\end{array}\right)$ and we get four linearly independent exponential solutions

$$
v_{+} e^{ \pm i k_{+} x}=\left(\begin{array}{c}
1 \\
i
\end{array}\right) e^{ \pm i k_{+} x}, \quad v_{-} e^{ \pm i k_{-} x}=\left(\begin{array}{r}
1 \\
-i
\end{array}\right) e^{ \pm i k_{-} x} .
$$


Now we can solve the equation (A. 3). First we rewrite it using the representations (4.9) and (4.8) for the operator $\mathbf{C}$,

$$
\left(\begin{array}{rr}
-\lambda & -\frac{d^{2}}{d x^{2}}+\omega \\
\frac{d^{2}}{d x^{2}}-\omega & -\lambda
\end{array}\right)\left(\begin{array}{l}
R_{11}(\lambda, x, y) \\
R_{21}(\lambda, x, y)
\end{array}\right)=\delta(x-y)\left(\begin{array}{l}
1 \\
0
\end{array}\right)+\delta(x)\left(\begin{array}{cc}
0 & a \\
-a-b & 0
\end{array}\right)\left(\begin{array}{l}
R_{11}(\lambda, 0, y) \\
R_{21}(\lambda, 0, y)
\end{array}\right)
$$

Let us consider $y>0$ for the concreteness. Then the RHS vanishes in the open intervals $(-\infty, 0),(0, y)$ and $(y, \infty)$. Hence, for the parameter $\lambda$ outside the cuts $C_{ \pm}$, the solution admits the representation

$$
R_{I}(\lambda, x, y)= \begin{cases}A_{+} e^{-i k_{+} x} v_{+}+A_{-} e^{-i k_{-} x} v_{-}, & x<0, \\ B_{+}^{-} e^{-i k_{+} x} v_{+}+B_{-}^{-} e^{-i k_{-} x} v_{-}+B_{+}^{+} e^{i k_{+} x} v_{+}+B_{-}^{+} e^{i k_{-} x} v_{-}, & 0<x<y, \\ C_{+} e^{i k_{+} x} v_{+}+C_{-} e^{i k_{-} x} v_{-}, & x>y\end{cases}
$$

since by (A. 8), the exponent $e^{-i k_{ \pm} x}$ decays for $x \rightarrow-\infty$, and similarly, $e^{i k_{ \pm} x}$ decays for $x \rightarrow \infty$. Next we need eight equations to calculate the eight constants $A_{+}, \ldots, C_{-}$. We have two continuity equations and two jump conditions for the derivatives at the points $x=0$ and $x=y$. These four vector equations give just eight scalar equations for the calculation.

Continuity at $x=y: R_{I}(y-0, y)=R_{I}(y+0, y)$, i.e.

$$
B_{-}^{-} v_{+} / e_{+}+B_{-}^{-} v_{-} / e_{-}+B_{+}^{+} v_{+} e_{+}+B_{-}^{+} v_{-} e_{-}=C_{+} v_{+} e_{+}+C_{-} v_{-} e_{-},
$$

where $e_{ \pm}:=e^{i k_{ \pm} y}$. It is equivalent to

$$
\left\{\begin{array}{l}
B_{+}^{-} / e_{+}+B_{+}^{+} e_{+}=C_{+} e_{+}, \\
B_{-}^{-} / e_{-}+B_{-}^{+} e_{-}=C_{-} e_{-} .
\end{array}\right.
$$

Continuity at $x=0: R_{I}(-0, y)=R_{I}(+0, y)$, i.e.

$$
A_{+} v_{+}+A_{-} v_{-}=B_{+}^{-} v_{+}+B_{-}^{-} v_{-}+B_{+}^{+} v_{+}+B_{-}^{+} v_{-}
$$

that is equivalent to

$$
\left\{\begin{array}{l}
A_{+}=B_{+}^{-}+B_{+}^{+}, \\
A_{-}=B_{-}^{-}+B_{-}^{+} .
\end{array}\right.
$$

Jump at $x=y: R_{I}^{\prime}(y+0, y)=R_{I}^{\prime}(y-0, y)+\left(\begin{array}{r}0 \\ -1\end{array}\right)$, where prime denotes the derivative in $x$. Substituting (A. 10), we get

$$
\begin{aligned}
& i k_{+} C_{+} v_{+} e_{+}+i k_{-} C_{-} v_{-} e_{-}= \\
& -i k_{+} B_{+}^{-} v_{+} / e_{+}-i k_{-} B_{-}^{-} v_{-} / e_{-}+i k_{+} B_{+}^{+} v_{+} e_{+}+i k_{-} B_{-}^{+} v_{-} e_{-}+\left(\begin{array}{r}
0 \\
-1
\end{array}\right)
\end{aligned}
$$


Noting that

$$
\left(\begin{array}{r}
0 \\
-1
\end{array}\right)=\frac{v_{+}-v_{-}}{2} i
$$

we get

$$
\left\{\begin{array}{c}
i k_{+} C_{+} e_{+}=-i k_{+} B_{+}^{-} / e_{+}+i k_{+} B_{+}^{+} e_{+}+\frac{i}{2}, \\
i k_{-} C_{-} e_{-}=-i k_{-} B_{-}^{-} / e_{-}+i k_{-} B_{-}^{+} e_{-}-\frac{i}{2} .
\end{array}\right.
$$

After substituting of $C_{ \pm}$from (A. 11), the constants $B_{ \pm}^{+}$cancel and we get

$$
B_{+}^{-}=\frac{e_{+}}{4 k_{+}}, \quad B_{-}^{-}=-\frac{e_{-}}{4 k_{-}} .
$$

Jump at $x=0: R_{I}^{\prime}(+0, y)=R_{I}^{\prime}(-0, y)-\left(\begin{array}{cc}a+b & 0 \\ 0 & a\end{array}\right) R_{I}(-0, y)$. Substituting (A. 10), we get

$-i k_{+} B_{+}^{-} v_{+}-i k_{-} B_{-}^{-} v_{-}+i k_{+} B_{+}^{+} v_{+}+i k_{-} B_{-}^{+} v_{-}=-i k_{+} A_{+} v_{+}-i k_{-} A_{-} v_{-}-M\left(A_{+} v_{+}+A_{-} v_{-}\right)$, where $M$ is the matrix $\left(\begin{array}{cc}a+b & 0 \\ 0 & a\end{array}\right)$. Note that

$$
\left\{\begin{array}{l}
M v_{+}=\alpha v_{+}+\beta v_{-} \\
M v_{-}=\alpha v_{-}+\beta v_{+}
\end{array}, \quad \text { where } \quad \alpha=a+\frac{b}{2}, \quad \beta=\frac{b}{2} .\right.
$$

Then (A. 17) becomes

$$
\left\{\begin{array}{l}
-i k_{+} B_{+}^{-}+i k_{+} B_{+}^{+}=-i k_{+} A_{+}-A_{+} \alpha-A_{-} \beta \\
-i k_{-} B_{-}^{-}+i k_{-} B_{-}^{+}=-i k_{-} A_{-}-A_{+} \beta-A_{-} \alpha
\end{array}\right.
$$

Substituting here (A. 12), we get after cancellations,

$$
\left\{\begin{array}{l}
\left(2 i k_{+}+\alpha\right) B_{+}^{+}+\beta B_{-}^{+}=-\alpha B_{+}^{-}-\beta B_{-}^{-} \\
\beta B_{+}^{+}+\left(2 i k_{-}+\alpha\right) B_{-}^{+}=-\beta B_{+}^{-}-\alpha B_{-}^{-}
\end{array}\right.
$$

Hence, the solution is given by

$$
\left(\begin{array}{c}
B_{+}^{+} \\
B_{-}^{+}
\end{array}\right)=-\frac{1}{D}\left(\begin{array}{cc}
2 i k_{-}+\alpha & -\beta \\
-\beta & 2 i k_{+}+\alpha
\end{array}\right)\left(\begin{array}{cc}
\alpha & \beta \\
\beta & \alpha
\end{array}\right)\left(\begin{array}{c}
B_{+}^{-} \\
B_{-}^{-}
\end{array}\right),
$$

where $D$ is the determinant

$$
D:=\left(2 i k_{+}+\alpha\right)\left(2 i k_{-}+\alpha\right)-\beta^{2},
$$

and $B_{+}^{-}, B_{-}^{-}$are given by (A. 16). The formulas (A. 16) and (A. 19) imply

$$
B_{+}^{+}=\frac{1}{2 D}\left(-\frac{2 i k_{-} \alpha+\alpha^{2}-\beta^{2}}{2 k_{+}} e_{+}+i \beta e_{-}\right), \quad B_{-}^{+}=\frac{1}{2 D}\left(-i \beta e_{+}+\frac{2 i k_{+} \alpha+\alpha^{2}-\beta^{2}}{2 k_{-}} e_{-}\right) .
$$


Using the identities

$$
2 i k_{-} \alpha+\alpha^{2}-\beta^{2}=D-2 i k_{+} \alpha+4 k_{+} k_{-}, \quad 2 i k_{+} \alpha+\alpha^{2}-\beta^{2}=D-2 i k_{-} \alpha+4 k_{+} k_{-},
$$

let us rewrite (A. 21) as

$$
B_{+}^{+}=-\frac{e_{+}}{4 k_{+}}+\frac{1}{2 D}\left(\left(i \alpha-2 k_{-}\right) e_{+}+i \beta e_{-}\right), \quad B_{-}^{+}=\frac{e_{-}}{4 k_{-}}-\frac{1}{2 D}\left(i \beta e_{+}+\left(i \alpha-2 k_{+}\right) e_{-}\right) .
$$

Finally, the formulas (A. 10)-(A. 12), (A. 16) and (A. 22) give the first column $R_{I}(\lambda, x, y)$ of the resolvent for $y>0$ :

$$
R_{I}(\lambda, x, y)=\Gamma_{I}(\lambda, x, y)+P_{I}(\lambda, x, y)
$$

where

$$
\Gamma_{I}(\lambda, x, y)=\frac{1}{4 k_{+}}\left(e^{i k_{+}|x-y|}-e^{i k_{+}(|x|+|y|)}\right) v_{+}-\frac{1}{4 k_{-}}\left(e^{i k_{-}|x-y|}-e^{i k_{-}(|x|+|y|)}\right) v_{-},
$$

and

$$
\begin{aligned}
P_{I}(\lambda, x, y) & =\frac{1}{2 D}\left[\left(\left(i \alpha-2 k_{-}\right) e^{i k_{+}(|x|+|y|)}+i \beta e^{i\left(k_{+}|x|+k_{-}|y|\right)}\right) v_{+}\right. \\
& \left.-\left(i \beta e^{i\left(k_{-}|x|+k_{+}|y|\right)}+\left(i \alpha-2 k_{+}\right) e^{i k_{-}(|x|+|y|)}\right) v_{-}\right]
\end{aligned}
$$

Calculation of second column The second column is given by similar formulas with the vector $\left(\begin{array}{l}1 \\ 0\end{array}\right)$ instead of $\left(\begin{array}{l}0 \\ 1\end{array}\right)$ in (A. 9). Then $\left(\begin{array}{r}0 \\ -1\end{array}\right)$ in (A. 13) is changed by $\left(\begin{array}{l}1 \\ 0\end{array}\right)$. Respectively, (A. 14) is changed by

$$
\left(\begin{array}{l}
1 \\
0
\end{array}\right)=\frac{v_{-}+v_{+}}{2} .
$$

Hence, we have now change $i / 2$ by $1 / 2$ in the first equation of (A. 15) and $-i / 2$ by $1 / 2$ in the second one. Respectively, (A. 16) for the second column reads

$$
B_{+}^{-}=-\frac{i e_{+}}{4 k_{+}}, \quad B_{-}^{-}=-\frac{i e_{-}}{4 k_{-}} .
$$

Then the second column $R_{I I}(\lambda, x, y)$ of the resolvent reads:

$$
R_{I I}(\lambda, x, y)=\Gamma_{I I}(\lambda, x, y)+P_{I I}(\lambda, x, y)
$$

where

$$
\Gamma_{I I}(\lambda, x, y)=-\frac{i}{4 k_{+}}\left(e^{i k_{+}|x-y|}-e^{i k_{+}(|x|+|y|)}\right) v_{+}-\frac{i}{4 k_{-}}\left(e^{i k_{-}|x-y|}-e^{i k_{-}(|x|+|y|)}\right) v_{-},
$$

and

$$
\begin{aligned}
P_{I I}(\lambda, x, y) & =\frac{i}{2 D}\left[\left(-\left(i \alpha-2 k_{-}\right) e^{i k_{+}(|x|+|y|)}+i \beta e^{i\left(k_{+}|x|+k_{-}|y|\right)}\right) v_{+}\right. \\
& \left.+\left(i \beta e^{i\left(k_{-}|x|+k_{+}|y|\right)}-\left(i \alpha-2 k_{+}\right) e^{i k_{-}(|x|+|y|)}\right) v_{-}\right]
\end{aligned}
$$

Note, that if $y<0$ we get the same formulas. 


\section{A2 The poles of the resolvent}

The poles of the resolvent correspond to the roots of the determinant (A. 20),

$$
D(\lambda):=\alpha^{2}+2 i \alpha\left(k_{+}+k_{-}\right)-4 k_{+} k_{-}-\beta^{2}=0 .
$$

with $k_{ \pm}$as in (A. 7)-(A. 8). Thus $D(\lambda)$ is an analytic function on $\mathbb{C} \backslash \mathcal{C}_{-} \cup \mathcal{C}_{+}$. Since there are two possible values for the square roots in $k_{ \pm}$there is a corresponding four-sheeted function $\tilde{D}(\lambda)$ analytic on a four sheeted cover of $\mathbb{C}$ which is branched over $\mathcal{C}_{-}$and $\mathcal{C}_{+}$. We call the sheet defined by (A. 8) the physical sheet.

We will reduce the equation (A. 23) to the solution of two successive quadratic equations. These can be solved explicitly but the process involves squaring and thus actually produces zeros of the function $\tilde{D}(\lambda)$ rather than of $D(\lambda)$. Therefore we will then have to check whether or not the roots do actually lie on the physical sheet.

Step i)

Denote by $\sigma=k_{+}+k_{-}$. Then

$$
\sigma^{2}=2 k_{+} k_{-}-2 \omega
$$

by (A. 7), hence (A. 23) gives the first quadratic equation:

$$
\alpha^{2}+2 i \alpha \sigma-2\left(\sigma^{2}+2 \omega\right)-\beta^{2}=0 .
$$

Rewrite it as

$$
\sigma^{2}-i \alpha \sigma=\frac{\alpha^{2}-\beta^{2}}{2}-2 \omega=: \delta
$$

Finally,

$$
\sigma=\frac{i \alpha}{2} \pm \sqrt{\delta-\frac{\alpha^{2}}{4}}
$$

where the root is choosen arbitrarily.

Further let us express the roots in $\omega$. Since $a=2 \sqrt{\omega}, \alpha=a+b / 2, \beta=b / 2$ then substituting $\delta$ from (A. 25), we obtain

$$
\delta-\frac{\alpha^{2}}{4}=\frac{\alpha^{2}}{4}-\frac{\beta^{2}}{2}-2 \omega=\frac{(a+b / 2)^{2}}{4}-\frac{b^{2}}{8}-\frac{a^{2}}{2}=-\frac{a^{2}}{4}-\frac{b^{2}}{16}+\frac{a b}{4}=-\frac{1}{16}(2 a-b)^{2}<0 .
$$

Now (A. 26) reads

$$
\sigma=\frac{i \alpha}{2} \pm \frac{i}{4}(2 a-b)=\frac{i}{4}[(2 a+b) \pm(2 a-b)]=i \gamma_{j}, j=1,2,
$$

where $\gamma_{j} \in \mathbb{R}$, and

$$
\gamma_{1}=a=a\left(C^{2}\right), \quad \gamma_{2}=b / 2=a^{\prime}\left(C^{2}\right) C^{2}
$$

Step ii)

It remains to calculate the correponding spectral parameter $\lambda$. First, the quadratic equation (A. 24) implies by (A. 27) that

$$
4\left(k_{+} k_{-}\right)^{2}=\left(2 \omega+\sigma^{2}\right)^{2}=\left(2 \omega-\gamma_{j}^{2}\right)^{2}, j=1,2 .
$$

On the other hand,

$$
k_{+} k_{-}=\sqrt{-\omega+i \lambda} \sqrt{-\omega-i \lambda},
$$


hence (A. 29) gives the second quadratic equation

$$
4\left(\omega^{2}+\lambda^{2}\right)=\left(2 \omega-\gamma_{j}^{2}\right)^{2} .
$$

Therefore,

$$
\lambda^{2}=\frac{\left(2 \omega-\gamma_{j}^{2}\right)^{2}-4 \omega^{2}}{4}=-\frac{\gamma_{j}^{2}\left(4 \omega-\gamma_{j}^{2}\right)}{4} .
$$

Finally, we obtain four roots

$$
\lambda_{j}=i \frac{\gamma_{j}}{2} \sqrt{4 \omega-\gamma_{j}^{2}}
$$

where $j \in\{1,2\}$ and the square root can takes two opposite values.

Corollary A.1 The four-sheeted function $\tilde{D}(\lambda)$ has the following roots (zeros):

i) $j=1$ gives $\lambda_{1}=0$ since $4 \omega=a^{2}=\gamma_{1}$.

ii) If $\left|\gamma_{2}\right|<2 \sqrt{\omega}$, then both $j=2$ roots $\pm i\left|\lambda_{2}\right|$ are pure imaginary.

iii) If $\left|\gamma_{2}\right|>2 \sqrt{\omega}$, then both $j=2$ roots $\pm\left|\lambda_{2}\right|$ are real: one positive and one negative.

Remark A.2 Note that a priori we can meet the wrong sign of $\operatorname{Im} k_{ \pm}$squaring (A. 30) which is why the above calculation yields roots of $\tilde{D}(\lambda)$ rather than the physical branch $D(\lambda)$. Since the formulas for the resolvent involve only $D(\lambda)$ it is important to know which of these are actually roots of $D(\lambda)$ and also to know the multiplicities. This is done in the next two sections.

\section{A3 Discrete spectrum $\lambda=0$}

In order to check that the roots of $\tilde{D}(\lambda)$ given in Corollary A.1 are actually roots of $D(\lambda)$ it suffices to check explicitly that $D(\lambda)$ vanishes (with the assumption that we are on the physical branch defined by $\operatorname{Im} k_{ \pm}>0$ for $\lambda \in \mathbb{C} \backslash \mathcal{C}_{ \pm}$.

For $j=1$ we have $\gamma=\gamma_{1}=a=2 \sqrt{\omega}$ and then $\lambda_{1}=0$. For $j=2$ we have $\gamma=\gamma_{2}=a^{\prime} C^{2}$. If $\left|\gamma_{2}\right|=2 \sqrt{\omega}$ ( equivalently $\left|a^{\prime}\right|=a / C^{2}$ ) or $\gamma_{2}=0$ ( equivalently $a^{\prime}=0$ ), we have $\lambda_{2}=0$.

Let us check that $\lambda=0$ is a root of $D(\lambda)$ :

$$
D(0)=\alpha^{2}-\beta^{2}+2 i \alpha 2 i \sqrt{\omega}+4 \omega=(a+b / 2)^{2}-b^{2} / 4-2(a+b / 2) a+a^{2}=0
$$

since $k_{ \pm}=i \sqrt{\omega}$. Now let us compute $D^{\prime}(\lambda)$ :

$$
D^{\prime}(\lambda)=i \alpha\left(\frac{i}{\sqrt{-\omega+i \lambda}}+\frac{-i}{\sqrt{-\omega-i \lambda}}\right)-\left(\frac{2 i}{\sqrt{-\omega+i \lambda}} \cdot \sqrt{-\omega-i \lambda}+\frac{-2 i}{\sqrt{-\omega-i \lambda}} \cdot \sqrt{-\omega+i \lambda}\right) .
$$

Hence $D^{\prime}(0)=0$ and $\lambda=0$ is the root of $D(\lambda)$ of multiplicity at least 2. Further calculation shows that the Taylor series for $D$ near zero takes the form:

$$
D(\lambda)=\left(\frac{1}{\omega}-\frac{b}{4 \omega^{3 / 2}}\right) \lambda^{2}+O\left(\lambda^{4}\right) .
$$

Therefore $\lambda=0$ is the root of $D(\lambda)$ of multiplicity 4 if and only if $b=4 \sqrt{\omega}$, i.e. $a^{\prime}=a / C^{2}$, and we have proved the following lemma:

Lemma A.3 If $a^{\prime}=a / C^{2}$ then $\lambda=0$ is a root of the determinant $D(\lambda)$ with multiplicity 4 , otherwise $\lambda=0$ is a root of the determinant $D(\lambda)$ with multiplicity 2. 


\section{A4 Nonzero discrete spectrum}

Now let us check whether the roots $\lambda=\lambda_{2} \neq 0$ corresponding $\gamma=\gamma_{2} \notin\{0, \pm 2 \sqrt{\omega}\}$ lie on the physical branch. We analyze two different cases: $0<\left|\gamma_{2}\right|<2 \sqrt{\omega}$ and $\left|\gamma_{2}\right|>2 \sqrt{\omega}$.

I. The case $\mathbf{0}<\left|\gamma_{\mathbf{2}}\right|<\mathbf{2} \sqrt{\omega}$ (equivalently $0<\left|a^{\prime}\right|<a / C^{2}$ ).

Since $4 \omega-\gamma_{2}^{2}>0$, the corresponding roots $\lambda_{2}$ are pure imaginary by (A. 31). Moreover, $\left|\lambda_{2}\right| \leq \omega$. Indeed, (A. 31) implies

$$
\omega^{2}-\left|\lambda_{2}\right|^{2}=\omega^{2}+\gamma_{2}^{4} / 4-\gamma_{2}^{2} \omega=\left(\omega-\gamma_{2}^{2} / 2\right)^{2} \geq 0
$$

Hence $-\omega \mp i \lambda_{2} \leq 0$ and $k_{ \pm}$are pure imaginary with nonnegative imaginary part, that is

$$
k_{+} k_{-} \leq 0 \text { and } \operatorname{Im}\left(\mathrm{k}_{+}+\mathrm{k}_{-}\right)>0 .
$$

The equations (A. 29) and (A. 24) imply

$$
\left|k_{+} k_{-}\right|=\frac{1}{4}\left|a^{2}-2\left(a^{\prime}\right)^{2} C^{4}\right|, \quad\left(k_{+}+k_{-}\right)^{2}=-2 \omega+2 k_{+} k_{-}=-\frac{a^{2}}{2}+2 k_{+} k_{-} .
$$

In order to obtain $k_{+} k_{-}$and $k_{+}+k_{-}$from the last two equations we have to divide the set $0<\left|a^{\prime}\right|<a / C^{2}$ onto three subsets:

$$
\left(-a / C^{2}, a / C^{2}\right) \backslash\{0\}=\left(-a / C^{2},-a / \sqrt{2} C^{2}\right] \cup\left(\left(-a / \sqrt{2} C^{2}, a / \sqrt{2} C^{2}\right) \backslash\{0\}\right) \cup\left[\frac{a}{\sqrt{2} C^{2}}, \frac{a}{C^{2}}\right) .
$$

1) First consider the case $a^{\prime} \in\left[\frac{a}{\sqrt{2} C^{2}}, \frac{a}{C^{2}}\right.$ ). Then (A. 33) and (A. 34) imply

$$
\begin{gathered}
k_{+} k_{-}=\frac{1}{4}\left(a^{2}-2\left(a^{\prime}\right)^{2} C^{4}\right) . \\
\left(k_{+}+k_{-}\right)^{2}=-\frac{a^{2}}{2}+\frac{a^{2}}{2}-\left(a^{\prime}\right)^{2} C^{4}=-\left(a^{\prime}\right)^{2} C^{4}, \\
k_{+}+k_{-}=i a^{\prime} C^{2},
\end{gathered}
$$

and using (A. 23), we obtain

$$
\begin{gathered}
D\left(\lambda_{2}\right)=\left(a+a^{\prime} C^{2}\right)^{2}-\left(a^{\prime} C^{2}\right)^{2}+2 i\left(a+a^{\prime} C^{2}\right)\left(k_{+}+k_{-}\right)-4 k_{+} k_{-} \\
=a^{2}+2 a a^{\prime} C^{2}-2\left(a+a^{\prime} C^{2}\right) a^{\prime} C^{2}-a^{2}+2\left(a^{\prime}\right)^{2} C^{4}=0 .
\end{gathered}
$$

Note that each $\gamma_{2}$ defines two values $\lambda_{2}$ up to factor \pm 1 . If we replace $\lambda_{2}$ by $-\lambda_{2}, k_{+}$and $k_{-}$ change places and our calculation remains valid. Therefore, both values of $\lambda_{2}$ are roots of $D(\lambda)$.

2) Further consider $a^{\prime} \in\left(-\frac{a}{C^{2}},-\frac{a}{\sqrt{2} C^{2}}\right]$. In this case

$$
k_{+} k_{-}=\frac{1}{4}\left(a^{2}-2\left(a^{\prime}\right)^{2} C^{4}\right), \quad k_{+}+k_{-}=-i a^{\prime} C^{2} .
$$

Then we have

$$
D\left(\lambda_{2}\right)=a^{2}+2 a a^{\prime} C^{2}+2\left(a+a^{\prime} C^{2}\right) a^{\prime} C^{2}-a^{2}+2\left(a^{\prime}\right)^{2} C^{4}=4 a^{\prime} C^{2}\left(a+a^{\prime} C^{2}\right) \neq 0
$$


since $a^{\prime} \neq 0$ and $a^{\prime} \neq-a / C^{2}$. Therefore in this case both values of $\lambda_{2}$ are not the roots of $D(\lambda)$. 3) Finally consider $0<\left|a^{\prime}\right|<\frac{a}{\sqrt{2} C^{2}}$. Then (A. 33)-(A. 34 imply that

$$
\begin{gathered}
k_{+} k_{-}=-\frac{1}{4}\left(a^{2}-2\left(a^{\prime}\right)^{2} C^{4}\right)<0, \\
\left(k_{+}+k_{-}\right)^{2}=-a^{2}+\left(a^{\prime}\right)^{2} C^{4}<0, \\
k_{+}+k_{-}=i \sqrt{a^{2}-\left(a^{\prime}\right)^{2} C^{4}} .
\end{gathered}
$$

Then we have

$$
D\left(\lambda_{2}\right)=a\left(a+2 a^{\prime} C^{2}\right)-2\left(a+a^{\prime} C^{2}\right) \sqrt{a^{2}-\left(a^{\prime}\right)^{2} C^{4}}+a^{2}-2\left(a^{\prime}\right)^{2} C^{4} .
$$

To solve the equation $D\left(\lambda_{2}\right)=0$ with respect to $a^{\prime}$, divide the RHS of (A. 35) by $C^{4} \neq 0$ and denote $p=a / C^{2}>0$. Then we get the equation

$$
p^{2}+p a^{\prime}-\left(a^{\prime}\right)^{2}=\left(p+a^{\prime}\right) \sqrt{p^{2}-\left(a^{\prime}\right)^{2}}, 0<\left|a^{\prime}\right|<p / \sqrt{2} .
$$

Squaring both side of (A. 36), we get

$$
2\left(a^{\prime}\right)^{4}-p^{2}\left(a^{\prime}\right)^{2}=0
$$

The equation has no solutions for $0<\left|a^{\prime}\right|<p / \sqrt{2}$ and hence $D\left(\lambda_{2}\right)$ does not vanish.

Corollary A.4 i) $D\left(\lambda_{2}\right)=0$ if $a^{\prime} \in\left[\frac{a}{\sqrt{2} C^{2}}, \frac{a}{C^{2}}\right)$.

ii) $D\left(\lambda_{2}\right) \neq 0$ if $a^{\prime} \in\left(-\frac{a}{C^{2}}, \frac{a}{\sqrt{2} C^{2}}\right) \backslash\{0\}$.

II. The case $\left|\gamma_{\mathbf{2}}\right|>\mathbf{2} \sqrt{\omega}$ (equivalently $\left|a^{\prime}\right|>a / C^{2}$ ).

Since $4 \omega-\gamma_{2}^{2}<0$, the corresponding roots (A. 31) are real: $\lambda_{2}^{-}<0<\lambda_{2}^{+}, \lambda_{2}^{-}=-\lambda_{2}^{+}$. It is easy to prove that $k_{ \pm}$take the form:

$$
k_{ \pm}= \pm \mu+i \nu, \quad \nu>0 .
$$

Therefore

$$
k_{+} k_{-}=-\mu^{2}-\nu^{2}<0, \quad k_{+}+k_{-}=2 i \nu
$$

1) First consider the case $a^{\prime}>a / C^{2}$.Then by (A. 34) and (A. 37)

$$
k_{+} k_{-}=\frac{1}{4}\left(a^{2}-2\left(a^{\prime}\right)^{2} C^{4}\right), \quad\left(k_{+}+k_{-}\right)^{2}=-\left(a^{\prime}\right)^{2} C^{4}, \quad k_{+}+k_{-}=i a^{\prime} C^{2} .
$$

Therefore

$$
\begin{gathered}
D\left(\lambda_{2}\right)=a\left(a+2 a^{\prime} C^{2}\right)+2 i\left(a+a^{\prime} C^{2}\right)\left(k_{+}+k_{-}\right)-4 k_{+} k_{-}= \\
a\left(a+2 a^{\prime} C^{2}\right)-2\left(a+a^{\prime} C^{2}\right) a^{\prime} C^{2}-a^{2}+2\left(a^{\prime}\right)^{2} C^{4}=0
\end{gathered}
$$

and then $\lambda_{2}$ are real roots of $D(\lambda)$. Hence, the case $a^{\prime}>a / C^{2}$ is linearly unstable.

2) Further consider the case $a^{\prime}<-a / C^{2}$. Then

$$
\begin{gathered}
k_{+} k_{-}=\frac{1}{4}\left(a^{2}-2\left(a^{\prime}\right)^{2} C^{4}\right)<0, \quad k_{+}+k_{-}=-i a^{\prime} C^{2}, \\
D\left(\lambda_{2}\right)=a^{2}+2 a a^{\prime} C^{2}+2\left(a+a^{\prime} C^{2}\right) a^{\prime} C^{2}-a^{2}+2\left(a^{\prime}\right)^{2} C^{4}=4 a^{\prime} C^{2}\left(a+a^{\prime} C^{2}\right) \neq 0
\end{gathered}
$$

Therefore, in this case $\lambda_{2}$ are not roots of $D(\lambda)$. 
Corollary A.5 i) In the unstable case $a^{\prime}>a / C^{2}$ : both $\lambda_{2}$ are roots of $D(\lambda)$. ii) If $a^{\prime}<-a / C^{2}$ then neither of the $\lambda_{2}$ are roots of $D(\lambda)$.

Summarizing, we have proved the following result

Theorem A.6 i) If $a^{\prime} \in\left(-\infty, a /\left(\sqrt{2} C^{2}\right)\right)$ the only root of $D(\lambda)$ is $\lambda=0$ with multiplicity 2. ii) If $a^{\prime} \in\left[a / \sqrt{2} C^{2}, a / C^{2}\right.$ ), there are four roots of $D(\lambda)$ : zero (multiplicity two) and $\pm i\left|\lambda_{2}\right|$ (pure imaginary) with $\lambda_{2}$ as in (A. 31).

iii) If $a^{\prime}=+a / C^{2}$, the only root of $D(\lambda)$ is $\lambda=0$ multiplicity 4 .

iv) If $a^{\prime} \in\left(a / C^{2},+\infty\right)$, there are four roots of $D(\lambda)$ : zero (multiplicity two) and $\pm\left|\lambda_{2}\right|$ with $\lambda_{2}$ as in (A. 31). In particular there exists a positive root (linear instability).

Remark A.7 Imagine reducing $a^{\prime}$ starting from a value greater than a $/ C^{2}$. Initially there are two real roots, $\pm\left|\lambda_{2}\right|$, which approach zero as $a^{\prime} \rightarrow a / C^{2}$ from above, giving rise to an increase of the multiplicity of the $\lambda=0$ root to four when $a^{\prime}=a / C^{2}$. As $a^{\prime}$ is reduced further below $a / C^{2}$ these two roots reappear as a pair of conjugate pure imaginary roots which move from zero to $\pm i \omega$ as $a^{\prime}$ goes from $a / C^{2}$ to $a / \sqrt{2} C^{2}$. When $a^{\prime}=a / \sqrt{2} C^{2}$ these two roots touch the branch point (end of the continuous spectrum) and move onto an "unphysical" branch (on which the conditions (A.8) do not hold). As $a^{\prime}$ is reduced further these roots do not return to the physical branch and thus even when their magnitude becomes zero they do not coalesce with the physical $\lambda=0$ root to increase its multiplicity and most importantly the spectrum is pure continuous apart from zero for $a^{\prime}<a / \sqrt{2} C^{2}$.

\section{References}

[1] H. Berestycki, P.L. Lions, Nonlinear scalar field equations I;II, Arch. Rat. Mech. and Anal. 82 (1983), no.4, 313-345; 347-375.

[2] V.S. Buslaev, G.S. Perelman, On nonlinear scattering of states which are close to a soliton, pp. 49-63 in: Méthodes Semi-Classiques, Vol.2 Colloque International (Nantes, juin 1991), Asterisque 208 (1992).

[3] V.S. Buslaev, G.S. Perelman, Scattering for the nonlinear Schrödinger equation: states close to a soliton, St. Petersburg Math. J.4 (1993), 1111-1142.

[4] V.S. Buslaev, G.S. Perelman, On the stability of solitary waves for nonlinear Schrödinger equations, Amer. Math. Soc. Trans. (2) 164 (1995), 75-98.

[5] V.S. Buslaev, C. Sulem, On asymptotic stability of solitary waves for nonlinear Schrödinger equations, Ann. Inst. Henri Poincaré, Anal. Non Linéaire 20(2003), no.3, 419-475.

[6] S. Cuccagna, On asymptotic stability of ground states of NLS, Rev. Math. Phys. 15 (2003), 877-903.

[7] P.A. Deift, A.R. Its, X. Zhou, Long-time asymptotics for integrable nonlinear wave equations, pp. 181-204 in: A.S. Fokas,V.E. Zakharov,(ed.), Important Developments in Soliton Theory, Springer, Berlin, 1993.

[8] L.D.Faddeev, L.A. Takhtadzhyan,Hamiltonian Methods in the Theory of Solitons Springer, Bewrlin, 1987. 
[9] M. Grillakis, J. Shatah, W.A. Strauss, Stability theory of solitary waves in the presence of symmetry, I; II. J. Func. Anal. 74 (1987), no.1, 160-197; 94 (1990), no.2, 308-348.

[10] V. Imaikin, A.I. Komech, B. Vainberg, On scattering of solitons for the Klein-Gordon equation coupled to a particle, submitted to Comm. Math. Phys., 2005.

[11] A. Jensen, T. Kato, Spectral properties of Schrö dinger operators and time-decay of the wave functions, Duke Math. J.46 (1979), 583-611.

[12] E. Kirr, A. Zarnesku On the asymptotic stability of bound states in 2D cubic Schro" dinger equation, Comm. Math. Phys. 272 (2007), no. 2, 443-468.

[13] A.I. Komech, A.A. Komech, On existence of solutions for the Schrödinger equation coupled to a nonlinear oscillator, preprint, 2006, math.AP/0608780.

[14] A.I. Komech, A.A. Komech, On global attraction to solitary waves for the Klein-Gordon equation coupled to nonlinear oscillator, C. R., Math., Acad. Sci. Paris, 343, Issue 2, 15 July 2006, Pages 111-114.

[15] A.I. Komech, A.A. Komech, Global attractor for a nonlinear oscillator coupled to the Klein-Gordon field, accepted in Arch. Rat. Mech. Anal., 2006. arXiv:math.AP/0609013

[16] J. Miller, M. Weinstein, Asymptotic stability of solitary waves for the regularized long-wave equation, Comm. Pure Appl. Math. 49 (1996), no. 4, 399-441.

[17] C.S. Morawetz, W.A. Strauss, Decay and scattering of solutions of a nonlinear relativistic wave equation, Comm. Pure Appl. Math. 25 (1972), 1-31.

[18] R.L. Pego, M.I. Weinstein, On asymptotic stability of solitary waves, Phys. Lett. A 162 (1992), 263-268.

[19] R.L. Pego, M.I. Weinstein, Asymptotic stability of solitary waves, Commun. Math. Phys. 164 (1994), 305-349.

[20] C.A. Pillet, C.E. Wayne, Invariant manifolds for a class of dispersive, Hamiltonian, partial differential equations, J. Differ. Equations 141 (1997), No.2, 310-326.

[21] F. Riesz, B. Sz.-Nagy, Functional Analysis, Dover Publications, NY, 1990.

[22] I. Rodnianski, W. Schlag, A. Soffer, Dispersive analysis of charge transfer models, Commun. Pure Appl. Math. 58 (2005), no. 2, 149-216.

[23] E. Schrödinger, Quantisierung als eigenwertproblem, Ann. d. Phys. 81 (1926), 109.

[24] A. Soffer, M.I. Weinstein, Multichannel nonlinear scattering for nonintegrable equations, Comm. Math. Phys. 133 (1990), 119-146.

[25] A. Soffer, M.I. Weinstein, Multichannel nonlinear scattering for nonintegrable equations. II. The case of anisotropic potentials and data, J. Differential Equations 98 (1992), no. 2, 376-390.

[26] A. Soffer, M.I. Weinstein, Resonances, radiation damping and instability in Hamiltonian nonlinear wave equations, Invent. Math. 136 (1999), 9-74. 
[27] A. Soffer, M.I. Weinstein, Selection of the ground states for NLS equations, Rev. Math. Phys. 16 (2004), no. 8, 977-1071.

[28] D.M.A. Stuart, Modulational approach to stability of non-topological solitons, Journal de Mathematiques Pures et Appliqu'ees 80 (2001), no. 1, 51-83.

[29] T.-P. Tsai, H.-T. Yau, Asymptotic dynamics of nonlinear Schrödinger equations: resonance-dominated and dispersion-dominated solutions, Commun. Pure Appl. Math. 55 (2002), no.2, 153-216.

[30] M. Weinstein, Modulational stability of ground states of nonlinear Schrödinger equations, SIAM J. Math. Anal. 16 (1985), no. 3, 472-491.

[31] A. Zygmund, Trigonometric Series I, Cambridge, 1968. 